\title{
Influence of pH on Morphology and Structure during Hydrolytic Degradation of the Segmented GL- $b$-[GL-co-TMC-co-CL]-b-GL Copolymer
}

\author{
Yolanda Márquez ${ }^{1,2}$, Juan Carlos Martínez ${ }^{3}$, Pau Turon ${ }^{2}$, Lourdes Franco ${ }^{1}$ and \\ Jordi Puiggalí ${ }^{1, *}$
}

1 Chemical Engineering Department, Polytechnic University of Catalonia, Av. Diagonal 647, Barcelona E-08028, Spain; E-Mails: ymlyolanda@gmail.com (Y.M.); lourdes.franco@upc.edu (L.F.)

2 B. Braun Surgical S.A., Carretera de Terrassa 121, Barcelona 08191, Spain;

E-Mail: pau.turon@bbraun.com

3 ALBA Synchrotron Light Facility, Ctra BP 1413 km 3.3, Cerdanyola del Vallès, Barcelona 08290, Spain; E-Mail: guilmar@ cells.es

* Author to whom correspondence should be addressed; E-Mail: jordi.puiggali@upc.edu; Tel.: +34-93-401-5649.

Academic Editor: Jingwei Xie

Received: 24 July 2015 / Accepted: 8 September 2015 / Published: 15 September 2015

\begin{abstract}
Hydrolytic degradation in media having a continuous variation of $\mathrm{pH}$ from 2 to 12 was studied for a copolymer having two polyglycolide hard blocks and a middle soft segment constituted by glycolide, trimethylene carbonate, and $\varepsilon$-caprolactone units. The last units were susceptible to cross-linking reactions by $\gamma$ irradiation that led to an increase of the molecular weight of the sample. Nevertheless, the susceptibility to hydrolytic degradation was enhanced with respect to non-irradiated samples and consequently such samples were selected to analyze the degradation process through weight loss measurements and the evaluation of changes on molecular weight, morphology, and SAXS patterns. Results reflected the different hydrolytic mechanisms that took place in acid and basic media and the different solubilization of the degradation products. Thus, degradation was faster and solubilization higher in the basic media. In this case, fibers showed a high surface erosion and the formation of both longitudinal and deep circumferential cracks that contrasted with the peeling process detected at intermediate pHs (from 6 to 8 ) and the absence of longitudinal cracks at low pHs. SAXS measurements indicated that degradation was initiated through the
\end{abstract}


hydrolysis of the irregular molecular folds placed on the amorphous interlamellar domains but also affected lamellar crystals at the last stages. Subsequent heating processes performed with degraded samples were fundamental to reveal the changes in microstructure that occurred during degradation and even the initial lamellar arrangement. In particular, the presence of interfibrillar domains and the disposition of lamellar domains at different levels along the fiber axis for a determined cross-section were evidenced.

Keywords: absorbable sutures; glycolide copolymer; hydrolytic degradation; $\gamma$-irradiation; lamellar microstructure; small angle X-ray scattering

\section{Introduction}

Sutures were the first commercial use of biodegradable polymers as orthopedic devices and by now their use has been extended to most surgical fields [1]. Nowadays, most resorbable sutures are polyesters (homopolymers and copolymers) based on glycolide, lactide, trimethylene carbonate, $\varepsilon$-caprolactone, and $p$-dioxanone units. These synthetic polymers, with hydrolizable bonds in their main chain, provide clear advantages over natural polymers such as tailored properties and even predictable lot-to-lot uniformity. In fact, control over degradation rate and properties can be achieved by the appropriate selection of monomers, copolymer composition, and copolymer architecture (e.g., blocky or random distributions). In this way, factors like hydrophilicity, crystallinity, chain stiffness, and molecular weight can be easily controlled [2].

Poly(glycolide), the first and simplest polyester employed as a suture [3], renders materials with high crystallinity, high melting and glass transition temperatures, high strength and Young modulus, and high degradation rate. The polymer is commercialized as a braided material due to its high stiffness and consequently has some potential inconveniences associated to tissue drag, knot tie-down, and risk of infection compared to monofilament sutures [4-6]. Flexible suture threads that are processed in a monofilament form have been manufactured to avoid these problems. Some of them consist on glycolide/trimethylene carbonate copolymers (named as polyglyconates) and a segmented A-B-A architecture where A is a polyglycolide hard block and B a random soft segment. MaxonTM (Syneture) was one of the former monofilament synthetic suture based in glycolide that has been developed [7]. Therefore, detailed studies concerning synthesis, degradation, properties, and crystallization have been performed on such copolymers as model materials for suture applications [8-20].

Works about monofilament sutures having three (e.g., Monosyn ${ }^{\mathrm{TM}}[21]$, and Biosyn ${ }^{\mathrm{TM}}[22,23]$ ) and four components (e.g., Caprosyn ${ }^{\mathrm{TM}}$ [24]) are more scarce despite the incorporation of additional monomers may lead to polymers with different properties and degradation behavior. Specifically, Monosyn $^{\mathrm{TM}}$ is the segmented GL- $b$-[GL-co-TMC-co-CL]- $b$-GL copolymer constituted by 72,14 and $14 \mathrm{wt} \%$ of glycolide, trimethylene carbonate and $\varepsilon$-caprolactone units, respectively. The material has a soft segment that represents the $43 \mathrm{wt} \%$ of the sample and includes $\varepsilon$-caprolactone units. The high methylene content provided by them lead to a decrease on the degradation rate that may balance the opposite effect caused by the increase on the amorphous character. 
Exposure to gamma rays is a process often employed for microbial decontamination of medical devices since this irradiation procedure is economically feasible for a large-scale terminal sterilization. Gamma irradiation is highly penetrating and ionizing events also activate numerous chemical reactions that can be useful to modify material properties. Basically, gamma irradiation may cause different effects on polymers: chain scission, crosslinking/grafting. The predominant process depends on the chemical nature of the polymer and the applied dose [25].

Polyester sutures exposed to gamma irradiation can experiment both a chain scission through the ester moiety and a crosslinking that involves radicals formed on the carbon atoms of methylene groups of the main chain [26]. Both crosslinking and degradation may occur simultaneously (i.e., gas release and increase of molecular weight may be observed together), although usually one process predominates. Obviously, the effect of these reactions on material properties is clearly different. Crosslinking increases the molecular weight, lowers the mobility of molecules, reduces creep, raises the tensile strength and increases the hardness and brittleness. Radiation induced degradation decreased the molecular weight as well as tensile, impact, shear strength, and elongation at break.

The predominant effect of $\gamma$ irradiation on polyglycolide is a chain scission that mainly leads to the unzipping of molecules and a faster loss of $M_{n}$ than $M_{w}$ [3]. Polyesters with a high number of methylene groups in the main chain are less susceptible to degradation since weakening of main chain bonds through ester resonance becomes less effective. Therefore, $\varepsilon$-caprolactone units belonging to the soft segment may be more susceptible to crosslinking than degradation. Note that segmented GL- $b$-[GL-co-TMC-co-CL]- $b$-GL could experiment a degradation through its hard segments and simultaneously a significant crosslinking through its soft segments that could give rise to an increase of the molecular weight. This is not the case of the bicomponent segmented copolymer GL- $b$-[GL-co-TMC]- $b$-GL, in which chain scission events are still predominant during irradiation. Nevertheless, the molecular weight decrease was lower than that observed for polyglycolide suggesting that trimethylene carbonate units were also susceptible to crosslinking reactions [27].

Molecular scission is expected to be more pronounced in the amorphous regions since recombination of formed radicals can be easier in the compact crystalline regions with immobile chain segments. Gamma irradiation should lead to a reduction of chain entanglement of polyglycolide segments in the amorphous phase and an open amorphous structure that facilitates hydrolytic degradation [27]. In the same way, polymers could become more susceptible to enzymatic attack after altering their physical and chemical structures by gamma irradiation [28].

Recent degradation studies on GL- $b$-[GL-co-TMC-co-CL]- $b$-GL revealed a complex process that could take place along longitudinal and lateral fiber directions depending on the degradation medium as also reported for other glycolide based sutures [27-29]. In all cases, disk morphologies were clearly detected in the last degradation stages, playing an important role the confinement of the amorphous soft segment between the two crystallizable polyglycolide hard blocks [29]. The present work insists on the morphological changes that took place during exposure of the gamma irradiated GL- $b$-[GL-co-TMC- $c o$-CL]- $b$-GL copolymer that was selected for its higher susceptibility to hydrolysis and its segmented architecture. To this end, media with a gradual variation of $\mathrm{pH}$ from 2 to 12 were considered and the lamellar morphology analyzed by means of small angle X-ray scattering (SAXS) of the exposed sutures before and after being submitted to a thermal annealing process that could enhance differences on the folding surfaces. 


\section{Experimental Section}

\subsection{Materials}

Commercially available sutures of GL- $b$-[GL-co-TMC-co-CL]- $b$-GL (Monosyn ${ }^{\text {TM }}$, USP 0) were kindly supplied by B. Braun Surgical, S.A. GL- $b$-[GL-co-TMC-co-CL]- $b$-GL samples were also exposed to a $\gamma$ irradiation dosage above $25 \mathrm{kGry}$ at a temperature of $25^{\circ} \mathrm{C}$ (i.e., slightly higher than the glass transition temperature). The segmented microstructure was attained by a two-step synthesis (Figure 1) where firstly a random soft segment was prepared and then used as initiator for the ring opening polymerization of glycolide.

a) $1^{\text {st }}$ Polymerization step: synthesis of soft segment

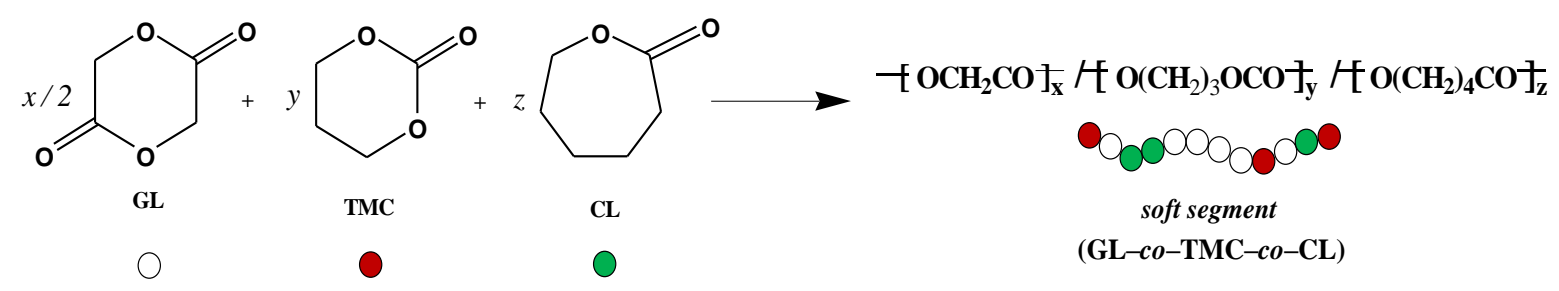

b) $2^{\text {nd }}$ Polymerization step: synthesis of segmented copolymer
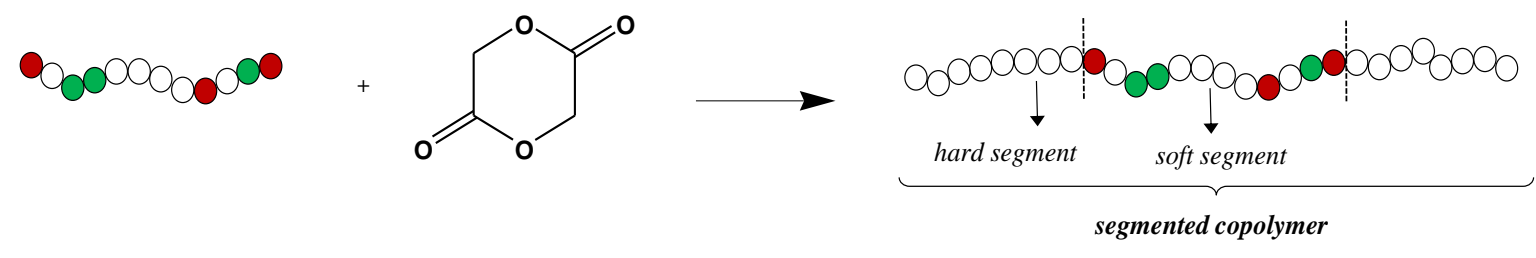

Figure 1. Two step synthesis of GL- $b$-[GL-co-TMC-co-CL]- $b$-GL showing the chemical structure of soft and hard segments.

\subsection{Hydrolytic Degradation}

In vitro hydrolytic degradation assays were carried out at a physiological temperature of $37^{\circ} \mathrm{C}$ and in the $\mathrm{pH}$ range from 2 to 12 using the Universal buffer (citrate-phosphate-borate/ $\mathrm{HCl}$ ) solution [30]. This was prepared by mixing $20 \mathrm{~mL}$ of a stock solution with $x \mathrm{~mL}$ of $0.1 \mathrm{M} \mathrm{HCl}$ and distilled water up to a volume of $100 \mathrm{~mL}$. The stock solution (1 L) contained $100 \mathrm{~mL}$ of citric acid, $100 \mathrm{~mL}$ of phosphoric acid, $3.54 \mathrm{~g}$ of boric acid and $343 \mathrm{~mL}$ of $1 \mathrm{M} \mathrm{NaOH}$. pHs 2, 3, 4, 5, 6, 7, 8, 9, 10, 11, and 12 were attained with 74.4, 56.9, 50.7, 45.4 39.7, 32.9, 28.1, 24.0, 18.1, 14.7, and 1.3 mL (i.e., the $x$ values) of the $\mathrm{HCl}$ solution, respectively.

Samples were kept under orbital shaking in bottles filled with $50 \mathrm{~mL}$ of the degradation medium and sodium azide $(0.03 \mathrm{wt} \%)$ to prevent microbial growth for selected exposure times. The samples were then thoroughly rinsed with distilled water, dried to constant weight under vacuum and stored over $\mathrm{P}_{4} \mathrm{O}_{10}$ before analysis. Weight retention, molecular weight, and calorimetric properties were then evaluated. Degradation studies were performed in quintuplicate and the given data corresponded to the average values. 


\subsection{Measurements}

Weight retention $\left(W_{r}\right)$ of the specimens was determined by the percentage ratio of weight after degradation $\left(W_{d}\right)$ to initial weight before degradation $\left(W_{0}\right)$ :

$$
W_{r}=W_{d} / W_{0} \times 100
$$

Molecular weights were estimated by size exclusion chromatography (GPC) using a liquid chromatograph (Shimadzu, model LC-8A, Tokyo, Japan) equipped with an Empower computer program (Waters). A PL HFIP gel column (Polymer Lab) and a refractive index detector (Shimadzu RID-10A, Tokyo, Japan) were employed. The polymer was dissolved and eluted in 1,1,1,3,3,3-hexafluoroisopropanol containing $\mathrm{CF}_{3} \mathrm{COONa}(0.05 \mathrm{M})$ at a flow rate of $1 \mathrm{~mL} / \mathrm{min}$ (injected volume $100 \mu \mathrm{L}$, sample concentration $2.0 \mathrm{mg} / \mathrm{mL}$ ). The number and weight average molecular weights were calculated using polymethyl methacrylate standards.

Calorimetric data were obtained by differential scanning calorimetry with a TA Instruments Q100 series with $T_{\text {zero }}$ technology and equipped with a refrigerated cooling system (RCS). A first heating run $\left(20{ }^{\circ} \mathrm{C} / \mathrm{min}\right.$ ) was performed to determine melting temperature and enthalpy, whereas a cooling run $\left(10^{\circ} \mathrm{C} / \mathrm{min}\right)$ after keeping the sample in the melt state for three minutes to erase the thermal history was carried out to determine crystallization data. Experiments were conducted under a flow of dry nitrogen with a sample weight of approximately $5 \mathrm{mg}$ and calibration was performed with indium. $T_{\text {zero }}$ calibration required two experiments: the first was performed without samples while sapphire disks were used in the second.

Optical micrographs were taken with a Zeiss Axioskop 40 Pol light polarizing microscope (Carl Zeiss, Oberkochen, Germany) equipped with a Zeiss AxiosCam MRC5 digital camera.

Scanning electron microscopy (SEM) was employed to examine the morphology of sutures after different times of exposure to the selected degradation media. Carbon coating was accomplished with a Mitec k950 Sputter Coater (fitted with a film thickness monitor k150x (Quorum Technologies Ltd., West Sussex, UK). SEM micrographs were obtained with a Zeiss Neon 40 EsB instrument (Carl Zeiss, Oberkochen, Germany).

Time resolved SAXS experiments were carried out at the NCD beamline (BL11) of the Alba synchrotron radiation light facility of Cerdanyola del Vallès (Catalunya). The beam was monochromatized to a wavelength of $0.1 \mathrm{~nm}$. Polymer samples were confined between Kapton films and then held on a Linkam THMS600 hot stage with temperature control within $\pm 0.1^{\circ} \mathrm{C}$. SAXS profiles were acquired during heating and cooling runs in time frames of $20 \mathrm{~s}$ and rates of $10{ }^{\circ} \mathrm{C} / \mathrm{min}$. The SAXS detector was calibrated with diffractions of a standard of a silver behenate sample. The diffraction profiles were normalized to the beam intensity and corrected considering the empty sample background.

\section{Results and Discussion}

\subsection{Hydrolytic Degradation of GL-b-[GL-co-TMC-co-CL]-b-GL in Different $p H$ Media}

Figure $2 \mathrm{a}$ shows the $\mathrm{pH}$ dependence of the degradation process evaluated through weight loss measurements during exposure to the different hydrolytic media. Degradation is characterized by sigmoidal curves with a short induction time, which is associated to the time required to render small 
soluble fragments able to diffuse from the fiber to the media, a period where an abrupt mass loss is observed and finally a period where degradation is slower due to the enrichment on more resistant fragments to the hydrolysis (e.g., crystalline regions). It is assumed that degradation initially occurs through random chain scissions that involved amorphous domains while a slower one-dimensional stepwise hydrolytic fragmentation is characteristic of the last stages where crystalline regions are more abundant [31].

a)

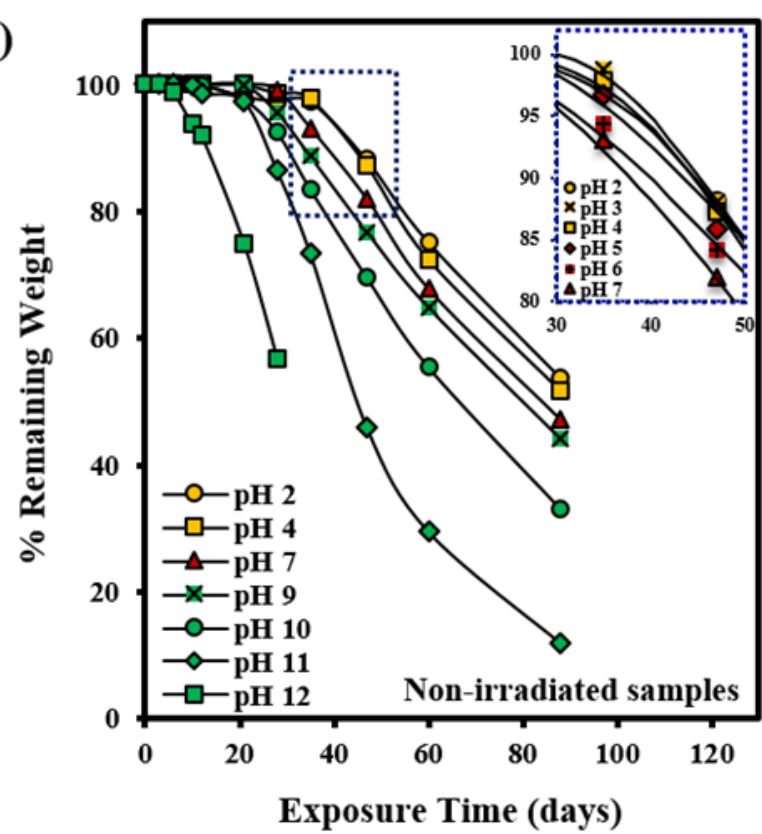

b)

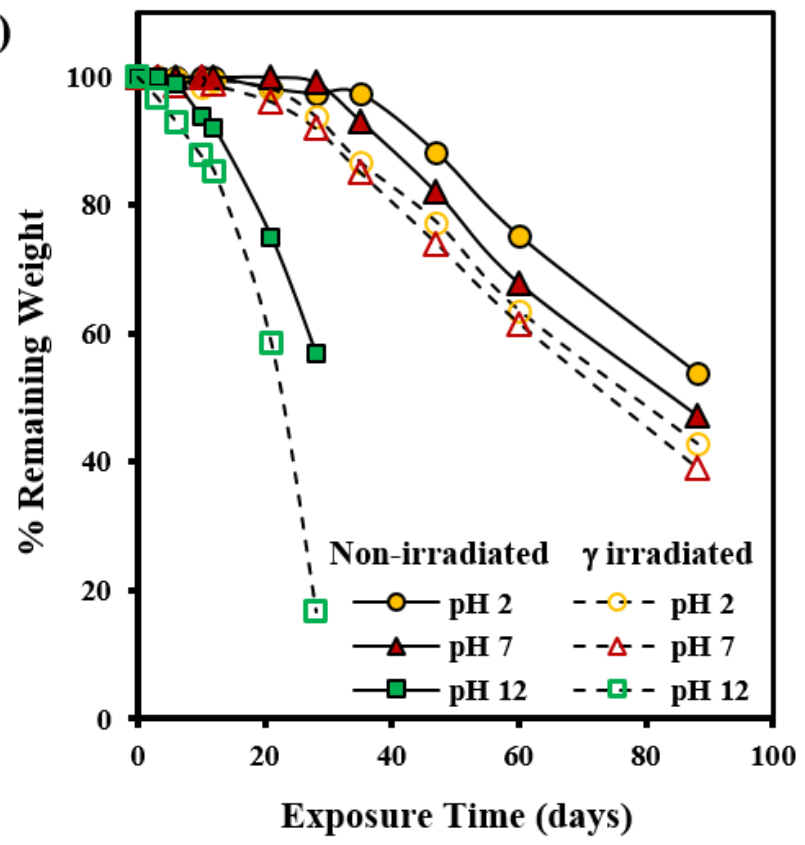

Figure 2. (a) Plot of remaining weight percentage versus exposure time to the different assayed hydrolytic degradation media for non-irradiated GL- $b$-[GL-co-TMC-co-CL]- $b$-GL samples. The inset shows a magnification for low $\mathrm{pH}$ media; (b) Comparison between degradation of non-irradiated and $\gamma$ irradiated GL- $b$-[GL-co-TMC-co-CL]- $b$-GL samples during exposure to different representative degradation media.

Results show a clear trend where induction time regularly decreases and the second step becomes narrower as the $\mathrm{pH}$ of the media increases. Nevertheless, differences were more pronounced by small increases on basicity than on acidity. Note for example that a weight loss of $40 \%$ required 41 and 27 days when $\mathrm{pH}$ increased from 11 to 12 , respectively, whereas 90 and 87 days were required for $\mathrm{pHs}$ of 2 and 3, respectively. Acid-catalyzed hydrolysis of esters is much slower at a given temperature than the alkali-catalyzed one giving rise also to a slower surface attack of the exposed samples [32]. It is well known that acid and alkaline hydrolysis take place through different mechanisms, being in the first case the process initiated by protonation of the single bonded oxygen of the ester group while in the second case the hydroxide ions attack the electron-deficient carbonyl carbons to form an intermediate anion.

Figure $2 \mathrm{~b}$ shows that degradation was clearly enhanced when samples were previously submitted to $\gamma$ irradiation. Basically, main differences on degradation profiles of irradiated and non-irradiated samples at a given $\mathrm{pH}$ correspond to the induction time. This was more significant in acid media and therefore a weight loss of $40 \%$ required 27 and 20 days at pH 12 while 90 and 73 days at $\mathrm{pH} 2$ for non-irradiated and $\gamma$ irradiated samples (Figure 3), respectively. 


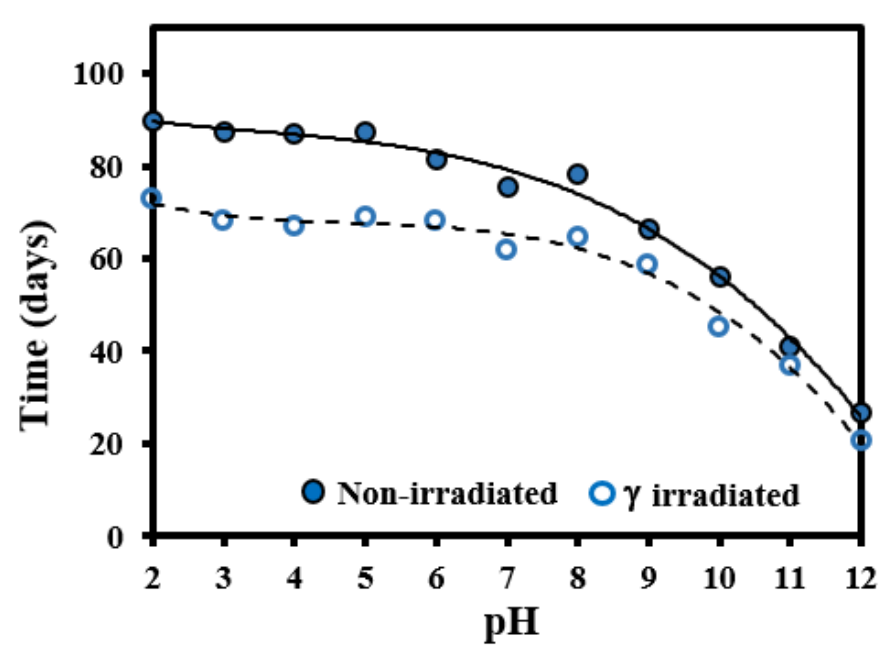

Figure 3. Plot of time required to get a weight loss of $40 \%$ for non-irradiated and $\gamma$ irradiated GL- $b$-[GL-co-TMC-co-CL]-b-GL samples exposed to different $\mathrm{pH}$ media.

Figure 4 shows the changes on the $\mathrm{pH}$ of the medium during the degradation process. A decrease was observed for basic media as a consequence of the release of degradation products with an acid nature. Basically, curves were characterized by an initial region where $\mathrm{pH}$ is constant, followed by a narrow (e.g., pH 12) or wide (e.g., $\mathrm{pH}$ 9) region where a significant $\mathrm{pH}$ decrease was observed due to the moderate buffer capacity of the media. Finally, a region where $\mathrm{pH}$ became again practically constant was detected. Logically, the magnitude of the $\mathrm{pH}$ change decreased and the second region shifted to higher times as the initial $\mathrm{pH}$ of the medium decreased, a feature that is in agreement with previously indicated weight loss measurements. In fact, times corresponding to the maximum slope of $\mathrm{pH}$ (Figure 4) and weight loss curves (Figure 2a) were similar. Comparison between pH curves of non-irradiated and $\gamma$ irradiated samples demonstrated again the faster degradation of the latter. Specifically, the maximum slope shifted for example from 30 to 23 days at $\mathrm{pH} 12$ and from 48 to 30 days at $\mathrm{pH} 9$.

Evolution of weight average molecular weight of non-irradiated and irradiated samples during exposure at the two extreme pHs (i.e., 2 and 11) is shown in Figure 5a. In this case curves were less influenced by the $\mathrm{pH}$ of the medium and were characterized by a rapid decrease during the first days of exposure until reaching a practically constant value at ca. 35 days. This can be interpreted as the minimum molecular size of insoluble degraded fragments. Note also that the initial molecular weight of the $\gamma$ irradiated sample was the highest (i.e., 102,400 with respect to $90,700 \mathrm{~g} / \mathrm{mol}$ ), suggesting that crosslinking reactions were produced during irradiation.

Figure $5 \mathrm{~b}$ shows the relative $M_{w}$ loss that allows for better comparison of the evolution of samples with different molecular sizes. It is clear that small differences could be found at the initial stages of degradation, being this enhanced for irradiated samples. The influence of $\mathrm{pH}$ is less clear but a faster decrease was observed at the beginning of exposure, probably as a consequence of the greater retention of small degraded molecules. Therefore, curves point out a variation on the degradation process that could obey a preferential degradation of the amorphous region at the beginning that is followed by a slower degradation of the edge of crystallites [33-35]. In fact, the first step can be associated to a quick random chain scission mechanism that follows a first-order kinetic, irrespective of the chain length, whereas a slower chain-end-scission mechanism can be expected when degradation affects the 
crystalline domains [29,36-39]. Note in Figure 5b the differences between the experimental curve and the simulated one considering a theoretical first order equation.

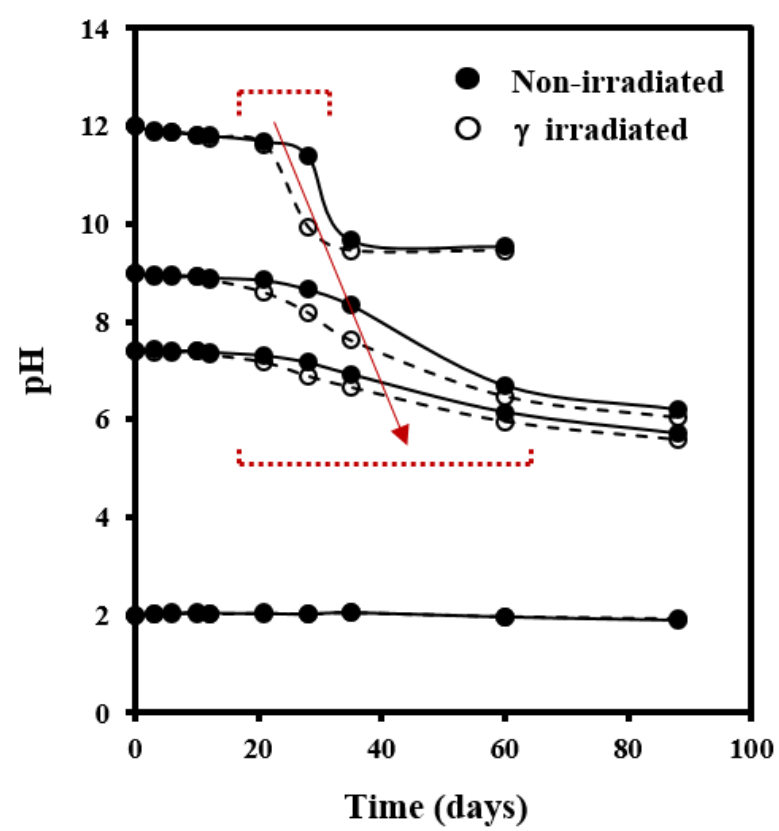

Figure 4. $\mathrm{pH}$ evolution of representative degradation media during exposure of non-irradiated (solid lines) and $\gamma$ irradiated (dashed lines) GL- $b$-[GL-co-TMC-co-CL]- $b$-GL samples.

a)

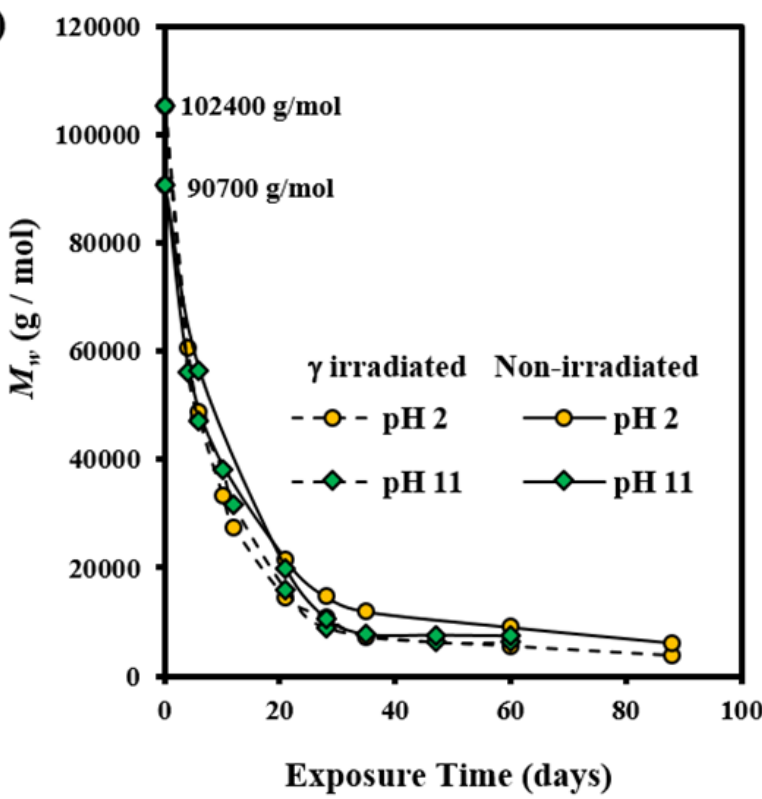

b)

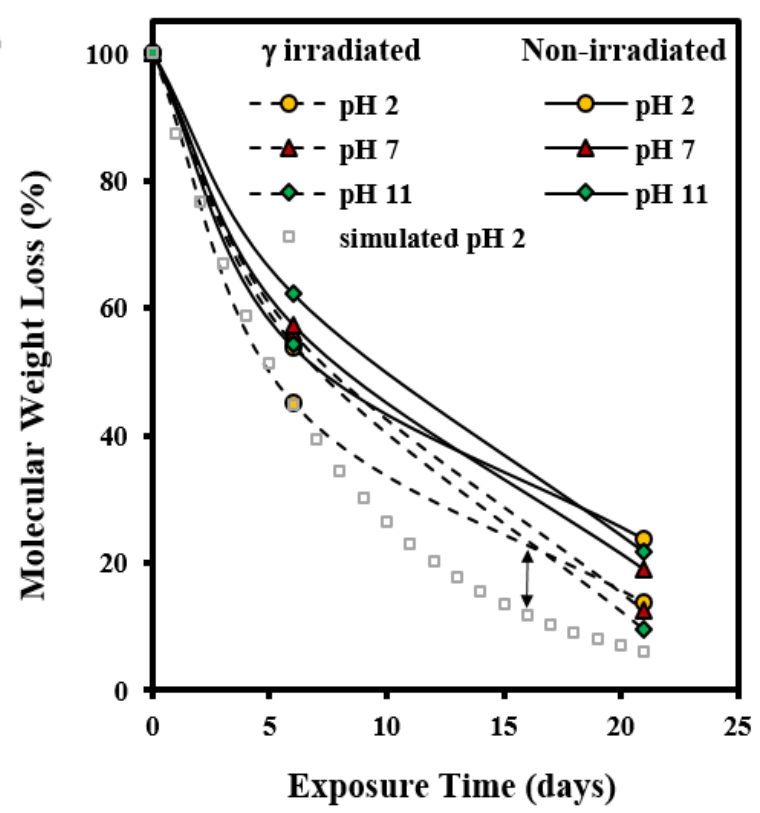

Figure 5. (a) Plots of the variation in the weight average molecular weight of a non-irradiated and a $\gamma$ irradiated GL- $b$-[GL-co-TMC-co-CL]- $b$-GL sample during exposure to a $\mathrm{pH} 12$ and $\mathrm{pH} 2$ degradation media; (b) Plots of the variation in the weight average molecular weight (percentage) of a non-irradiated and a $\gamma$ irradiated GL- $b$-[GL-co-TMC-co-CL]- $b$-GL sample during the first steps of exposure to representative degradation media. For the sake of completeness simulated data for a degradation following a first order equation is also drawn (dashed line) for the $\gamma$ irradiated sample exposed to $\mathrm{pH} 2$. 
Figure 6 shows typical DSC scans obtained during heating and cooling experiments, which demonstrated that samples were semicrystalline and able to easily crystallize from the melt state.

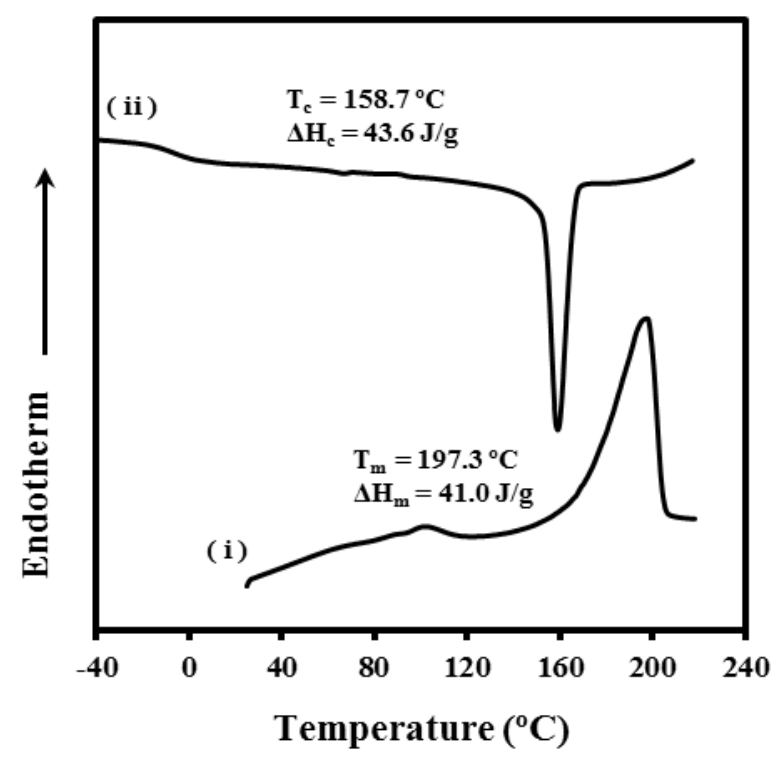

Figure 6. DSC traces obtained with the original $\gamma$ irradiated GL- $b$-[GL-co-TMC-co-CL]- $b$-GL sample. Traces correspond to the first heating run (i) and the cooling run after keeping the sample in the melt state for $3 \mathrm{~min}$ (ii).

Changes on thermal properties point also out the time required to produce a significant degradation. Thus, melting temperature abruptly decreased as well as the crystallization temperature when the minimum molecular weight was attained as shown in Figure $7 \mathrm{a}$ for the degradation in the $\mathrm{pH} 7$ representative medium. Therefore, lamellar crystals were sufficiently affected at the indicated degradation level to note a significant decrease on the melting temperature (e.g., from $200-198{ }^{\circ} \mathrm{C}$ to $191{ }^{\circ} \mathrm{C}$ ). Furthermore, differences were also detected between irradiated and non-irradiated samples, specifically the abrupt temperature changes occurred at lower exposure when samples were irradiated (i.e., 28 with respect to 21 days). Nevertheless, the effect of degradation could be better detected through the major difficulty to crystallize for the degraded samples. In this way, crystallization temperature decreased from an initial value of $165-160{ }^{\circ} \mathrm{C}$ to temperatures of 130 and $110{ }^{\circ} \mathrm{C}$ for non-irradiated and irradiated samples, respectively. The larger variation observed in the second case confirms again the greater susceptibility to degradation of samples after being $\gamma$ irradiated. Similar trends were observed from melting and crystallization enthalpies of degraded samples (Figure $7 \mathrm{~b}$ ). Thus, both enthalpies seem to reach a maximum value at the beginning of degradation as expected from a preferential attack to the amorphous region. Logically this maximum was observed at a lower exposure time when the $\gamma$ irradiated samples was considered. 
a)

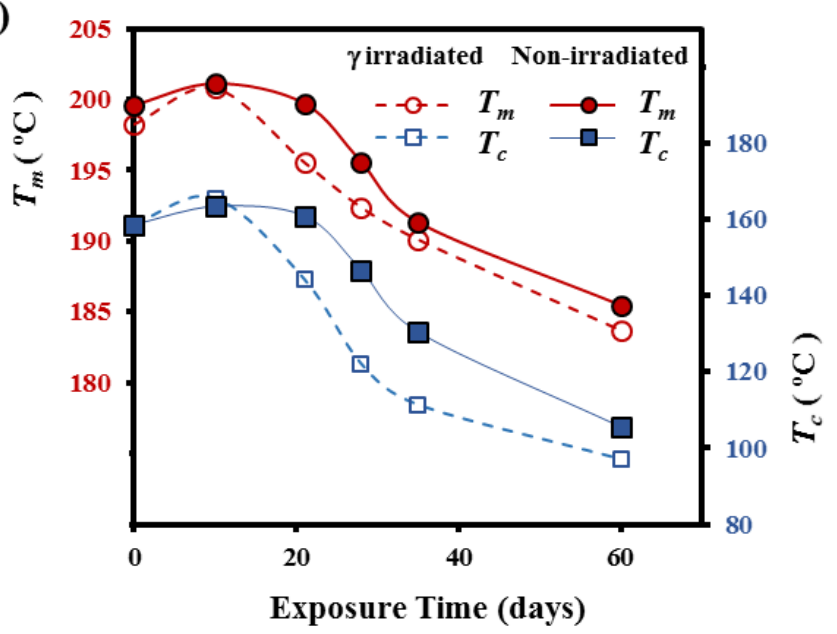

b)

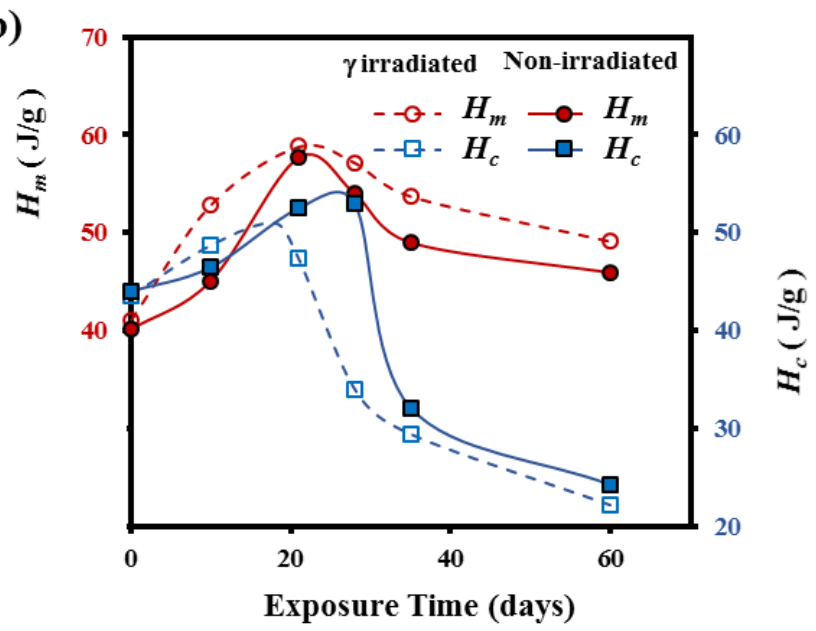

Figure 7. (a) Variation of melting and crystallization temperature and (b) melting and crystallization enthalpies of non-irradiated and $\gamma$ irradiated GL- $b$-[GL-co-TMC-co-CL]- $b$-GL samples during exposure time in a $\mathrm{pH} 7$ buffer medium.

\subsection{Morphological Changes during Hydrolytic Degradation of GL-b-[GL-co-TMC-co-CL]-b-GL in} Different $\mathrm{pH}$ Media

GL- $b$-[GL-co-TMC-co-CL]- $b$-GL oriented fibers exposed to a hydrolytic degradation process experience complex morphological changes as a consequence of different factors:

(1)Fibers are constituted by oriented lamellae and different amorphous regions which will be more susceptible to the hydrolysis process. In fact, oriented crystallites are embedded in an amorphous matrix, being possible to distinguish between interlamellar and interfibrilar amorphous regions (Figure 8a), according to previously postulated models [40,41]. The interlamellar domains alternate with lamellae in the direction of the fiber and possess the lowest molecular orientation and density since they are formed by molecular folds, tie chain segments between adjacent lamellar structures, and free chain ends. Therefore, these domains appear to be the most susceptible to hydrolysis. On the contrary, the interfibrilar domains may have a partial orientation and correspond to the regions placed on lateral sides of lamellae arranged in a fibrillar way. Degradation may therefore follow two different pathways: longitudinal and transversal depending on whether the hydrolysis proceeds mainly through the interfibrilar or interlamellar regions, respectively.

(2)The hydrolysis mechanism is different as previously indicated for acid and basic media. Differences not only concern the kinetic mechanism but also the capability to solubilize degradation products. In this way, retention may be significant in both the interlamellar and interfibrillar regions when an acidic medium is employed and therefore lateral and longitudinal diffusion of water molecules could be hindered. On the contrary, rapid solubilization of degradation products may enhance the surface erosion of exposed fibers in a basic medium.

(3)Morphology of fibers is not completely homogeneous due to a spinning process where the fiber surface is cooled faster than the core. Therefore, the shell layer should have different degree of molecular orientation and probably is constituted by a slightly different lamellar architecture (e.g. size of crystalline and amorphous domains). 
a)

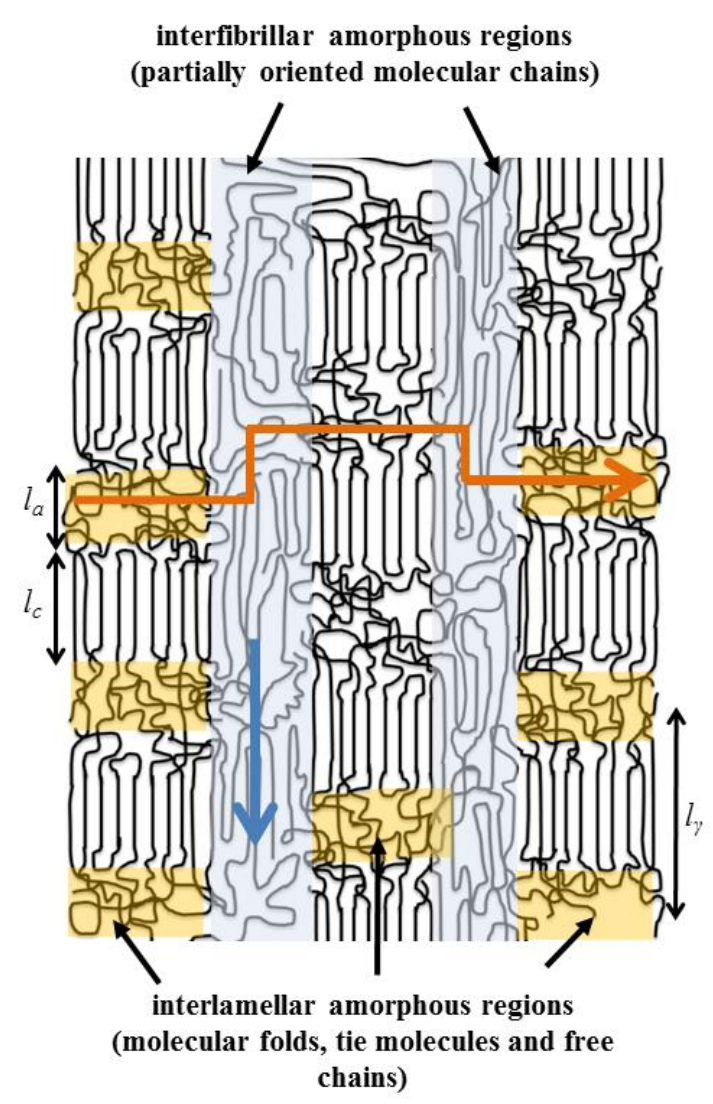

b) DEGRADED FIBER
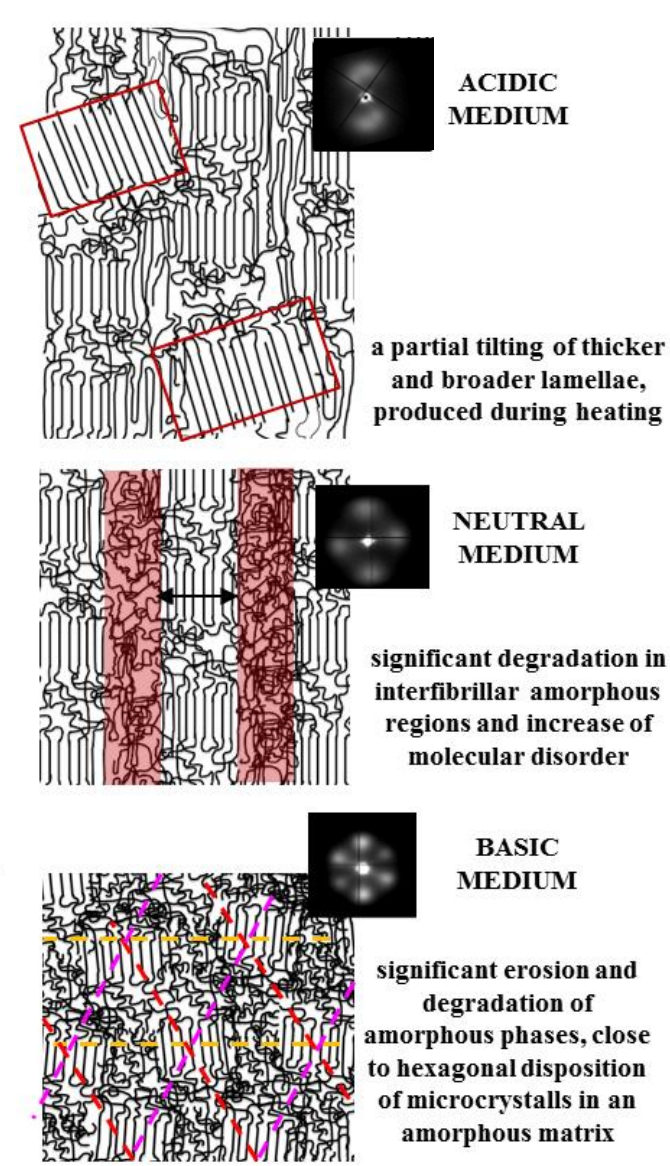

Figure 8. (a) Scheme showing the presence of oriented lamellar crystals embedded in interlamellar (yellow) and interfibrillar (blue) amorphous domains. Blue and orange arrows indicate the attack able to generate longitudinal and circumferential cracks, respectively; (b) Schemes showing the distinctive morphological changes that occurred during heating of samples previously degraded in acidic, neutral, and basic media. For the sake of completeness representative SAXS patterns taken during a heating scan are also shown.

Morphological inspection of fibers exposed to different $\mathrm{pH}$ media revealed a continuous evolution with pH and logically with time as shown in Figures 9-11. Morphological changes were similar for non-irradiated and $\gamma$ irradiated samples as shown in Figure 9 for a representative $\mathrm{pH}$ medium. However, in all cases changes proceeded faster when samples were exposed to irradiation in agreement with the above indicated accelerated degradation. Thus, longitudinal cracks and peeling were evident after exposure of the irradiated sample to a $\mathrm{pH} 7$ medium for 21 and 35 days, respectively, whereas the non-irradiated fiber was practically unaltered after 21 days. 35 and 47 days were required to appreciate cracks and peeling. Some features can be highlighted according to the optical and SEM micrographs obtained at neutral (Figures 9 and 12), acidic (Figures 10 and 12), and basic (Figures 11 and 12) pHs. 

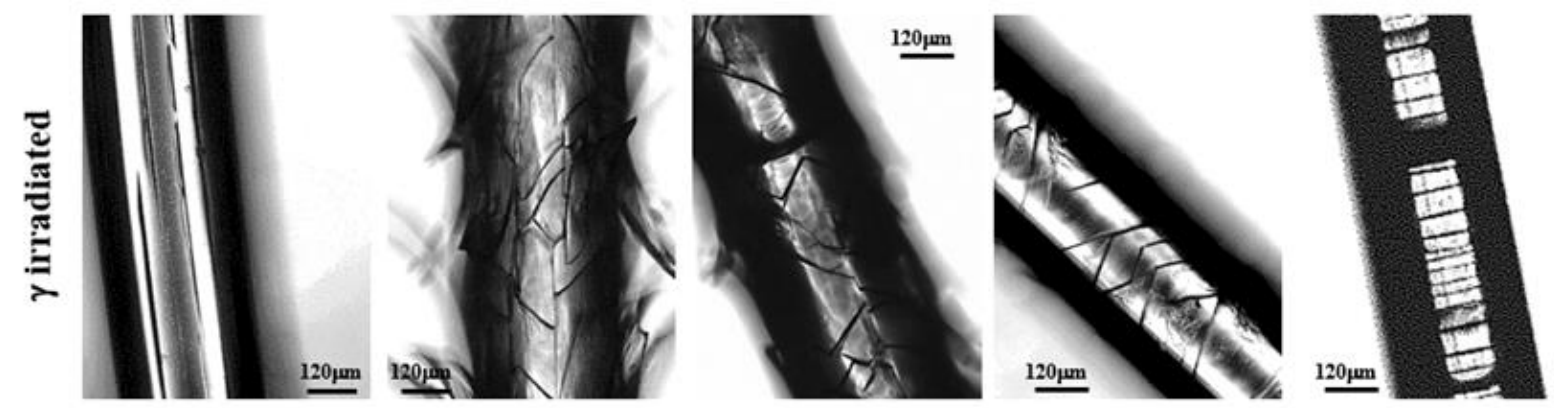

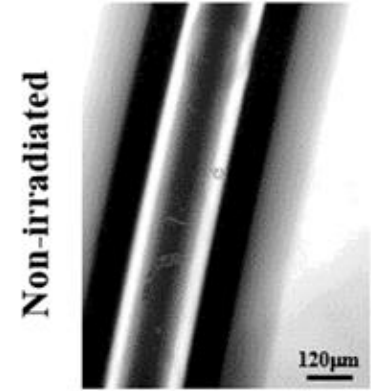

21 days

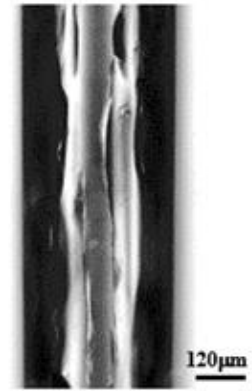

35 days

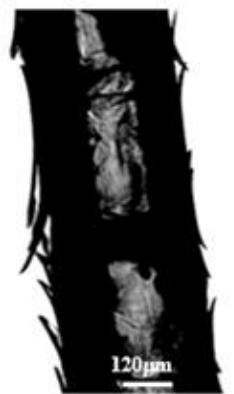

47 days

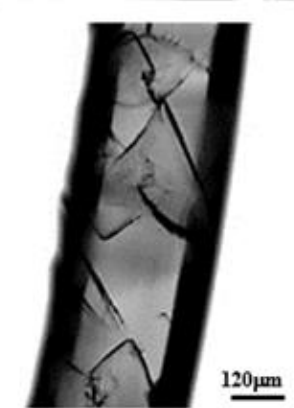

60 days

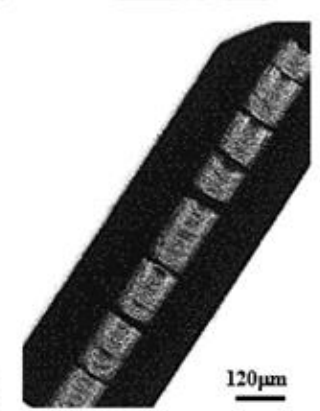

88 days

Figure 9. Optical micrographs showing the morphological changes of non-irradiated and $\gamma$ irradiated GL- $b$-[GL-co-TMC-co-CL]- $b$-GL samples during exposure for the indicated times to a $\mathrm{pH} 7$ buffer medium at $37^{\circ} \mathrm{C}$.

(1)Degradation in a neutral $\mathrm{pH}$ proceeds through three well differentiated steps. The first one corresponds to the development of straight and longitudinal cracks that evolve through the detachment of the skin layer. Microcracks that propagate circumferentially around the fiber axis are characteristic of the second step although they initially contribute to the peeling out process of the outermost skin. In this step microcracks are irregularly distributed along the fiber and are not completely extended along the circumferential perimeter. In fact, optical micrographs show the presence of irregular cracks that appear inclined with respect to the fiber axis. The last step is associated to the deep propagation of the initiated circumferential cracks through the cross-sectional planes where interlamellar amorphous domains more susceptible to the hydrolysis exist. At the end of this step, regularly distributed discs perpendicular to the fiber axis are evident in the optical and SEM micrographs.

(2)Peeling was not observed when the $\mathrm{pH}$ of the degradation medium was lower than 6 and furthermore the formation of longitudinal cracks was clearly hindered in these acidic media (Figure 10). Logically, degradation became slower as the $\mathrm{pH}$ decreased and in fact unaltered surfaces were observed after 21 days of exposure even for the irradiated sample. Circumferential and highly spaced cracks perpendicular to the fiber axis were detected at the first stages of degradation in the most acidic media (pHs 2 and 3) whereas irregular and inclined cracks were observed in the optical microscopy images taken at pHs between 4 and 6. Nevertheless, SEM micrographs revealed that these cracks had a zig-zag appearance. In all cases, additional cracks appeared at longer exposures, giving rise to irregular fissures that ultimately lead to the thinner and regular discs that were perfectly oriented perpendicular to the fiber axis. 
(3)Degradation in basic media showed the formation of deep longitudinal cracks at the beginning of exposure that lead to peeling only when $\mathrm{pH}$ was lower than 9. Circumferential cracks were also observed at the earlier degradation steps together with a clear erosion of the fiber surface, which can already be detected in the irradiated samples exposed for only 21 days in a pH 10 medium. SEM micrographs revealed also the formation of such circumferential cracks and the resulting wrinkled fiber surface. In fact, degradation in the high basic media was peculiar since deep transversal cracks that led to narrow discs were practically formed at the beginning of exposure. Note also that the high solubilization of degradation products caused the lineal and smooth fiber profile rapidly to evolve towards highly tortuous surfaces.
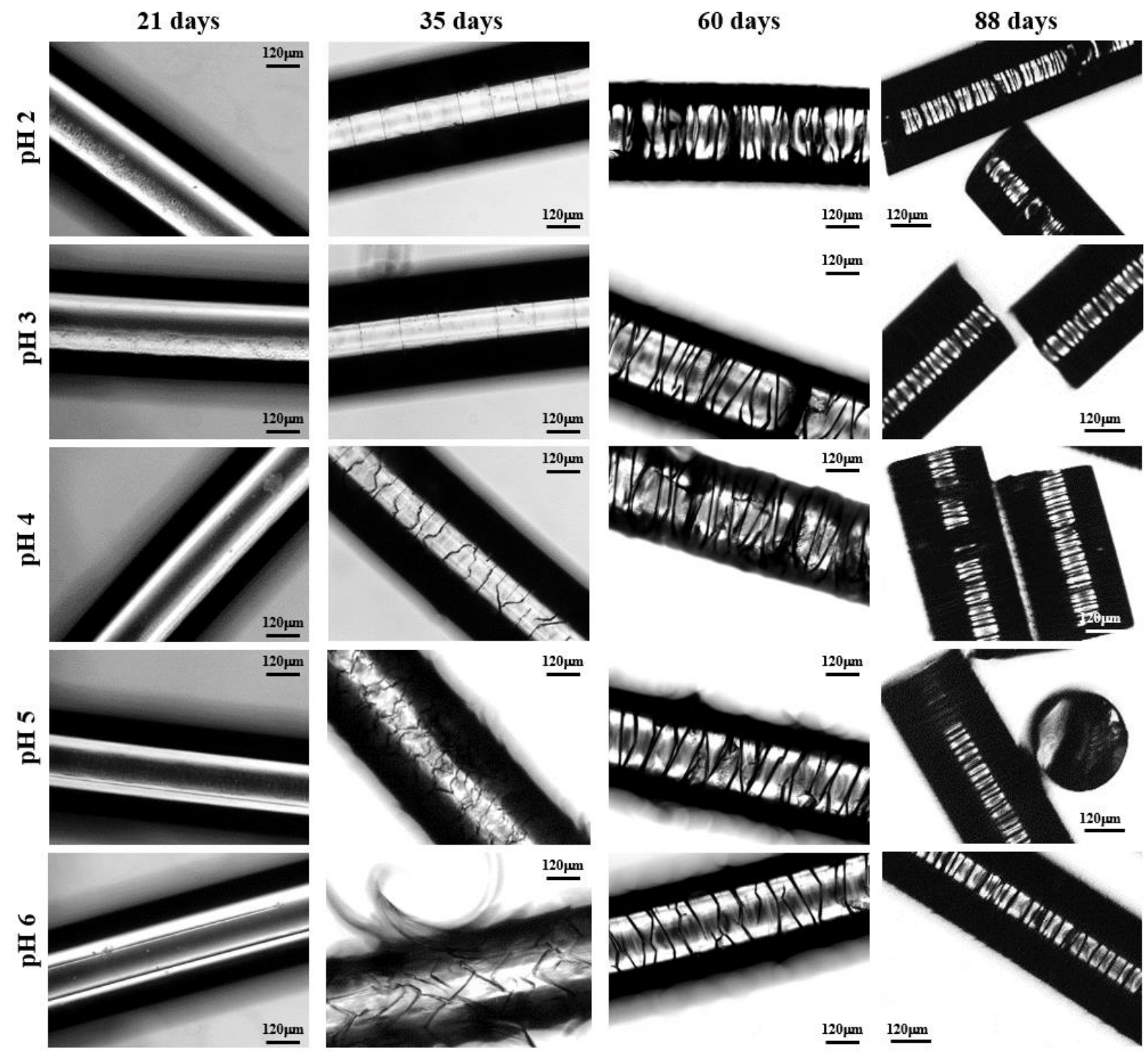

Figure 10. Optical micrographs showing the morphological changes of $\gamma$ irradiated GL- $b$-[GL-co-TMC-co-CL]- $b$-GL samples during exposure for the indicated times to the indicated acidic media at $37^{\circ} \mathrm{C}$. 


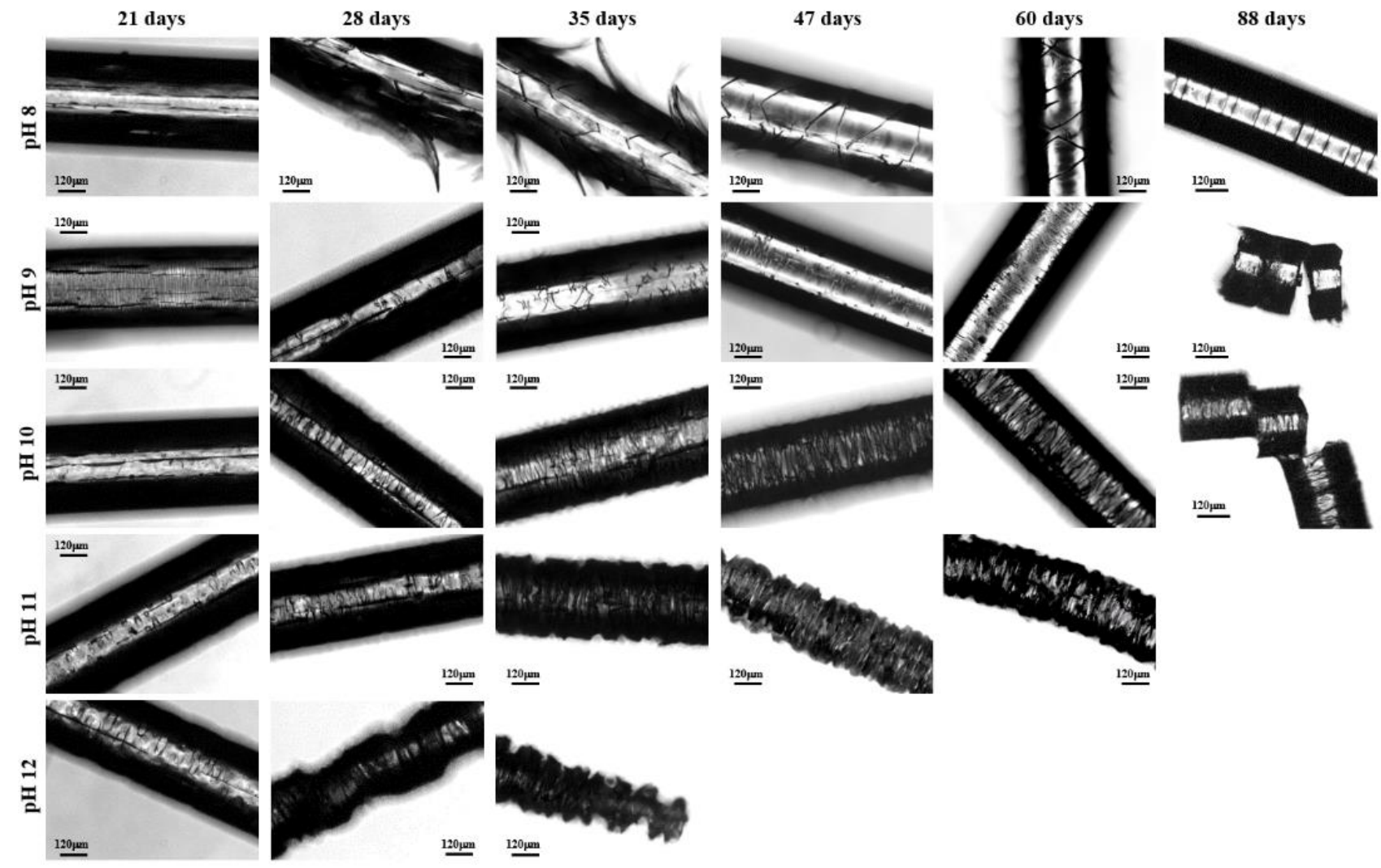

Figure 11. Optical micrographs showing the morphological changes of $\gamma$ irradiated GL- $b$-[GL-co-TMC-co-CL]- $b$-GL samples during exposure for the indicated times to the indicated basic media at $37^{\circ} \mathrm{C}$.
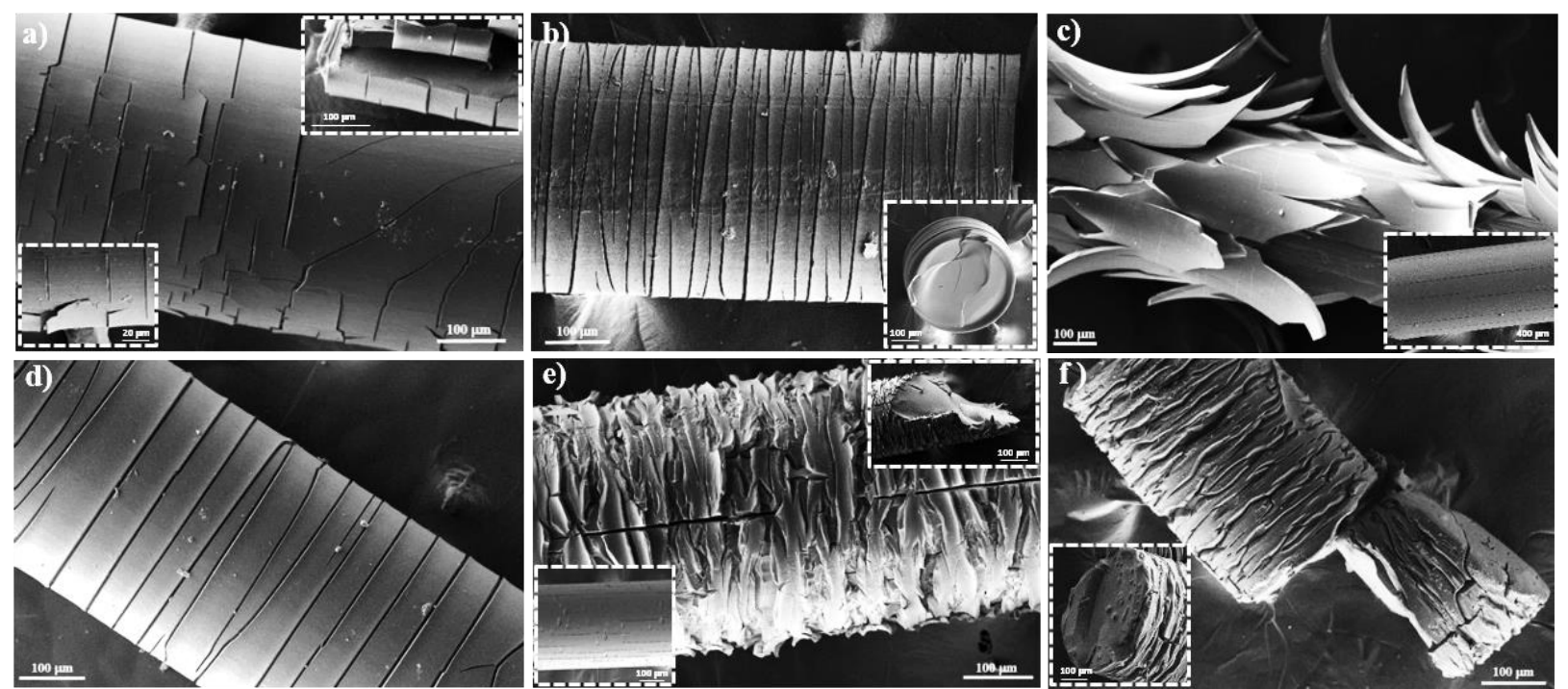

Figure 12. SEM micrographs showing the morphological changes of a $\gamma$ irradiated GL- $b$-(GL-co-TMC-co-CL)- $b$-GL sample during exposure to: (a) a pH 4 medium for 35 days (inset 55 days); (b) a pH 4 medium for 88 days (inset cross-section detail); (c) a pH 7 medium for 35 days (inset 21 days); (d) a pH 7 medium for 88 days; (e) a pH 10 medium for 35 days (insets 21 days and cross-section detail at 35 days) and (f) a pH 10 medium for 88 days (inset cross-section detail). 


\subsection{Changes on the Lamellar Parameters of GL-b-[GL-co-TMC-co-CL]-b-GL during Hydrolytic} Degradation in Different $\mathrm{pH}$ Media

Lamellar structure of the non-irradiated and $\gamma$ irradiated GL- $b$-[GL-co-TMC-co-CL]- $b$-GL samples gave rise to a strong meridional reflection in the SAXS pattern that corresponds to a $L_{B}$ spacing close to 8.3-7.6 nm (e.g., Figure 13a for the $\gamma$ irradiated sample). This high intensity suggests a large difference between the electronic density of amorphous and crystalline phases, which is in agreement with the tight packing of polyglycolide [42]. Slight differences on the degree of orientation can be detected, being in general lower after exposure to highly basic medium.

a)
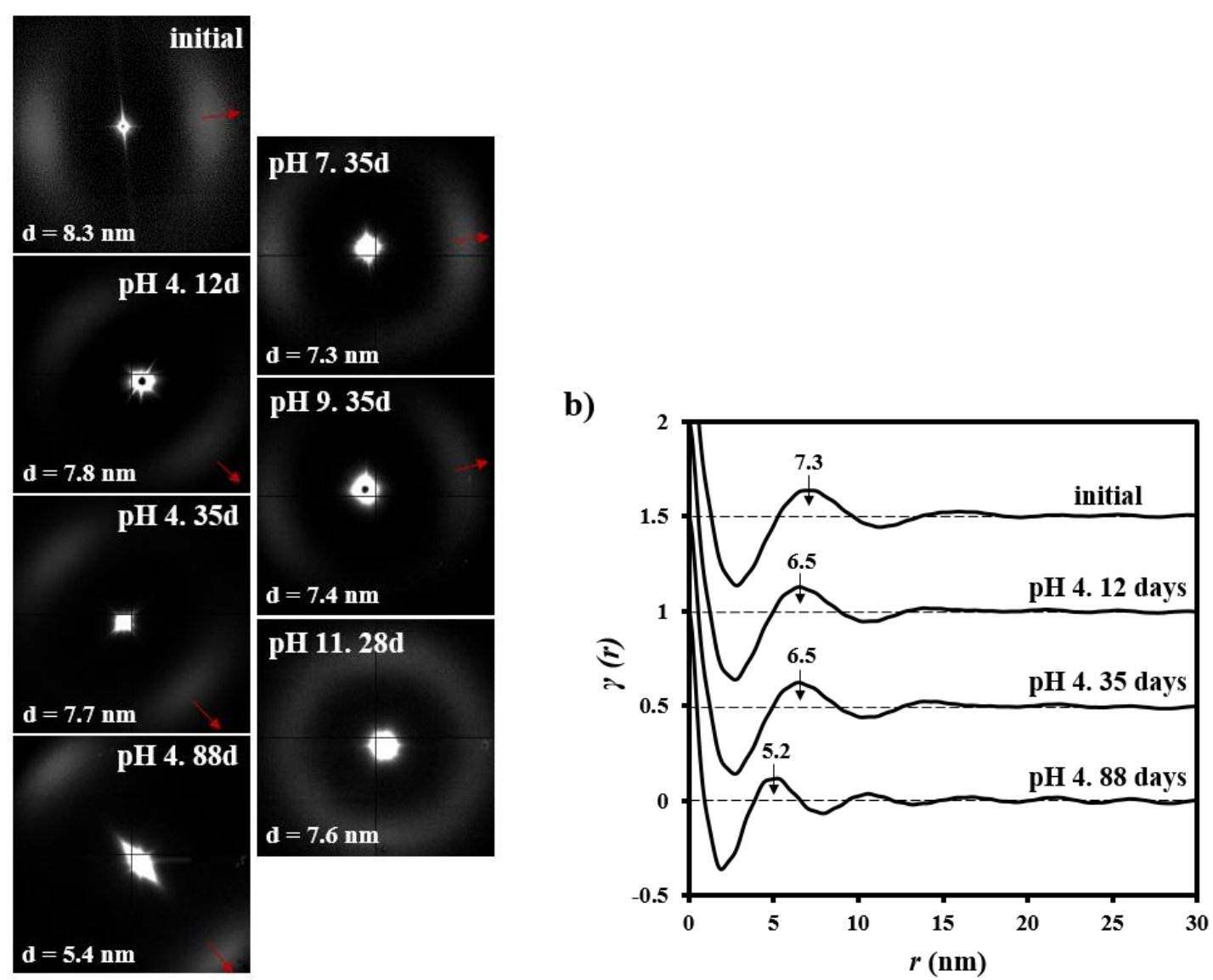

Figure 13. (a) SAXS patterns of the initial $\gamma$ irradiated GL- $b$-[GL-co-TMC-co-CL]- $b$-GL sample and after exposure to $\mathrm{pH} 4$ for 12, 35 and 88 days, $\mathrm{pH} 7$ for 35 days, $\mathrm{pH} 9$ for 35 days and $\mathrm{pH} 11$ for 28 days. Red arrows indicate the meridional fiber direction; (b) Comparison between correlation functions of $\gamma$ irradiated GL- $b$-[GL-co-TMC-co-CL]- $b$-GL sample for: initial sample, after exposure to $\mathrm{pH} 4$ for 12,35 , and 88 days.

Both non-irradiated and $\gamma$ irradiated samples exposed to the degradation media showed also the strong and oriented lamellar reflection (Figure 13 for representative $\gamma$ irradiated samples) even when fibers began to lose their integrity. More interestingly the lamellar spacing clearly decreased in the advanced degradation stages from its initial value (e.g., $8.3 \mathrm{~nm}$ for the irradiated sample) to a spacing close to $5.40 \mathrm{~nm}$ that was reached just before the fiber integrity was lost (e.g., Figure 13a for fibers exposed to a pH 4 medium during 88 days). On the contrary, slight variations on the lamellar spacing were detected at all 
assayed pHs during the period associated to the molecular weight decrease (i.e., 0-35 days), namely just before to attain the asymptotic molecular weight (e.g., Figure 13a for irradiated fibers exposed to pHs 4, 7,9 , and 11 , respectively). For example, a decrease from 8.3 to $7.7 \mathrm{~nm}$ was detected in this period at $\mathrm{pH}$ 4 (Figure 13a).

Characteristic lamellar parameters (i.e., long period, $L_{\gamma}$, amorphous layer thickness, $l_{a}$, and crystalline lamellar thickness, $l_{c}$ ) and crystallinity (i.e., crystallinity within the lamellar stacks, $X_{c}{ }^{S A X S}=l_{c} / L_{\gamma}$ ) were determined by means of the normalized one-dimensional correlation function [43], $\gamma(\mathrm{r})$ :

$$
\gamma(r)=\int_{0}^{\infty} q^{2} I(q) \cos (q r) d q / \int_{0}^{\infty} q^{2} I(q) d q
$$

where $I$ is the intensity and $q$ the scattering vector $([4 \pi / \lambda] \operatorname{sen} \theta)$.

SAXS data were collected within a limited angular range and consequently Vonk's model [44] and Porod's law were applied to perform extrapolations to low and high $q$ values.

Correlation functions of representative degraded samples are displayed in Figure 13b, whereas the determined morphological parameters and crystallinities are summarized in Table 1.

Table 1. Morphological parameters of $\gamma$ irradiated GL- $b$-[GL-co-TMC-co-CL]- $b$-GL samples during degradation in the indicated degradation media and exposure times.

\begin{tabular}{ccccccc}
\hline $\mathbf{p H}$ & Time $(\mathbf{D a y s})$ & $\boldsymbol{L}_{\boldsymbol{B}}(\mathbf{n m})$ & $\boldsymbol{L}_{\boldsymbol{\gamma}}(\mathbf{n m})$ & $\boldsymbol{l}_{\boldsymbol{c}}(\mathbf{n m})$ & $\boldsymbol{l}_{\boldsymbol{a}}(\mathbf{n m})$ & $\boldsymbol{X}_{\boldsymbol{c}} \boldsymbol{S A X S}^{\mathrm{AAS}}$ \\
\hline- & 0 & 8.3 & 7.3 & 5.3 & 2.0 & 0.73 \\
4 & 12 & 7.8 & 6.5 & 5.2 & 1.3 & 0.80 \\
4 & 35 & 7.7 & 6.5 & 5.2 & 1.3 & 0.80 \\
4 & 88 & 5.4 & 5.2 & 4.2 & 1.0 & 0.79 \\
7 & 35 & 7.3 & 6.4 & 5.1 & 1.3 & 0.80 \\
9 & 35 & 7.4 & 6.4 & 5.1 & 1.3 & 0.80 \\
11 & 28 & 7.6 & 6.6 & 5.2 & 1.4 & 0.80 \\
\hline
\end{tabular}

The one-dimensional correlation function is a relatively simple approximation and practicable method to derive the general structural differences of samples despite having some degree of orientation [45]. Additionally, it should be indicated that other methods have been developed to characterize more accurate samples with fiber symmetry (e.g., Chord Distribution Function [46,47]). The derived parameters indicate that degradation logically proceeded at the beginning of exposure through the molecular folds that constitute the interlamellar domains. Thus, $l_{c}$ remained practically constant (i.e., 5.2-5.1 nm), whereas the amorphous layer thickness decreased from 2.0 to $1.3 \mathrm{~nm}$ (i.e., a reduction close to $35 \%$ ). At the last stages of degradation the amorphous layer remained practically constant, being for example determined values close to $1.0 \mathrm{~nm}$ for $l_{a}$ (i.e., a reduction close to $50 \%$ ). At the same time the crystalline lamellar thickness started to decrease (i.e., a reduction of $21 \%$ corresponds to the observed change from 5.3 to $4.2 \mathrm{~nm}$ ), which means that hydrolysis and depolymerization affected the crystalline domains. In any case, the analysis demonstrates that the lamellar decrease was mainly caused by the disappearance of the amorphous layer as expected from a higher susceptibility to hydrolysis of the molecular folds placed on the lamellar surfaces.

Correlation functions revealed also that initial samples had a broad distribution of the layer widths of the crystal phase since $L_{\gamma}$ was higher than the long period determined from twice the value of the first 
minimum of the function (i.e., $5.6 \mathrm{~nm}$ respect to $7.3 \mathrm{~nm}$ ). However, homogeneity increased during the first degradation step that involved the hydrolysis of the amorphous layer (e.g., $5.6 \mathrm{~nm}$ and $6.5 \mathrm{~nm}$ were determined for samples exposed for 12 and 35 days at the $\mathrm{pH} 4$ medium). Logically, a wide distribution was again observed at the end when degradation affected the crystalline lamellar layers.

\subsection{Thermal Annealing of Degraded GL-b-[GL-co-TMC-co-CL]-b-GL Samples in Different $p H$}

Media: Repercussions on the Lamellar Morphology

The segmented polymer undergoes a lamellar reordering process on heating that causes an increase of the crystal thickness. Specifically, the irradiated sample had a change from an initial value of $8.3 \mathrm{~nm}$ to a spacing close to $20.8 \mathrm{~nm}$ that was attained just some degrees before fusion (Figure 14a). In addition, patterns also show how the corresponding arched reflections became highly oriented and adopted a circular shape, which evidenced an increase of the lamellar breadth and an irregular lamellar stacking.

Analysis of the correlation function (Figure 14b) demonstrates that the increase of the $L_{\gamma}$ interlamellar spacing (from $7.3 \mathrm{~nm}$ to $16.8 \mathrm{~nm}$ ) mainly corresponds to the regular increase of the crystalline lamellar thickness that changed from $5.3 \mathrm{~nm}$ to $13.8 \mathrm{~nm}$, whereas a lower variation was found for the amorphous layer (i.e., from 2.0 to $3.0 \mathrm{~nm}$ ). Nevertheless, the evolution of both spacing was proportional after achieving a temperature of $145{ }^{\circ} \mathrm{C}$ and $X_{c}^{S A X S}$ subsequently remained practically constant (i.e., close to 0.79-0.82).

a)

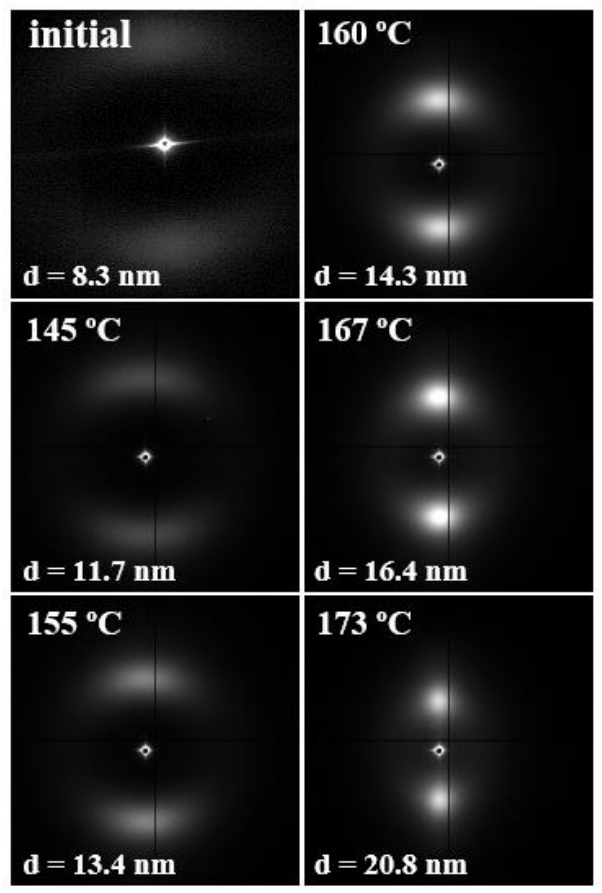

b)

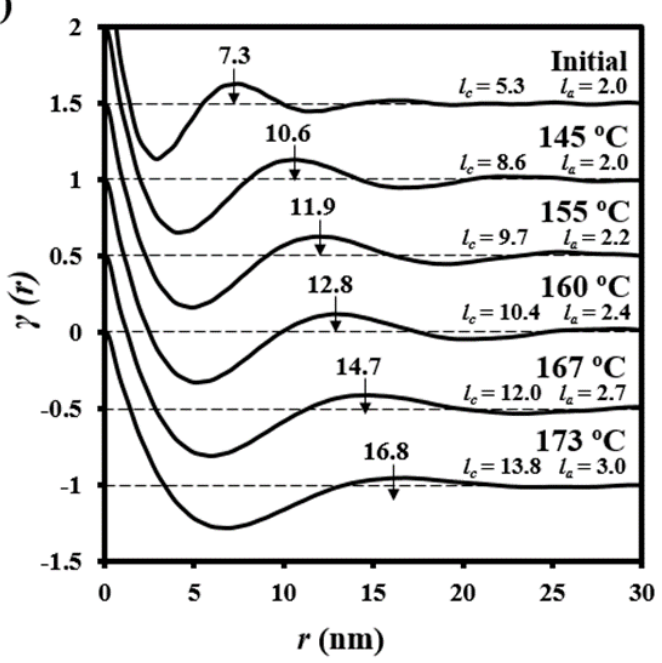

Figure 14. (a) SAXS patterns of the initial $\gamma$ irradiated GL- $b$-[GL-co-TMC-co-CL]- $b$-GL sample taken at representative temperatures during a heating run at $10{ }^{\circ} \mathrm{C} / \mathrm{min}$ (up); (b) Change on the correlation function during a heating run at $10{ }^{\circ} \mathrm{C} / \mathrm{min}$ of the initial $\gamma$ irradiated GL- $b$-[GL-co-TMC-co-CL]- $b$-GL sample. 
Lamellar microstructure is modified during hydrolytic degradation and consequently significant differences on the reordering process that occurs on heating should be expected not only respect the non-degraded sample but also between samples with different degrees of degradation or those exposed to different $\mathrm{pH}$ media. Figure 15 demonstrates the great differences on the evolution of SAXS patterns during heating for representative samples, being worthy of comment some observations that allow getting insight the initial fiber microstructure and how it was affected by degradation in the different media.

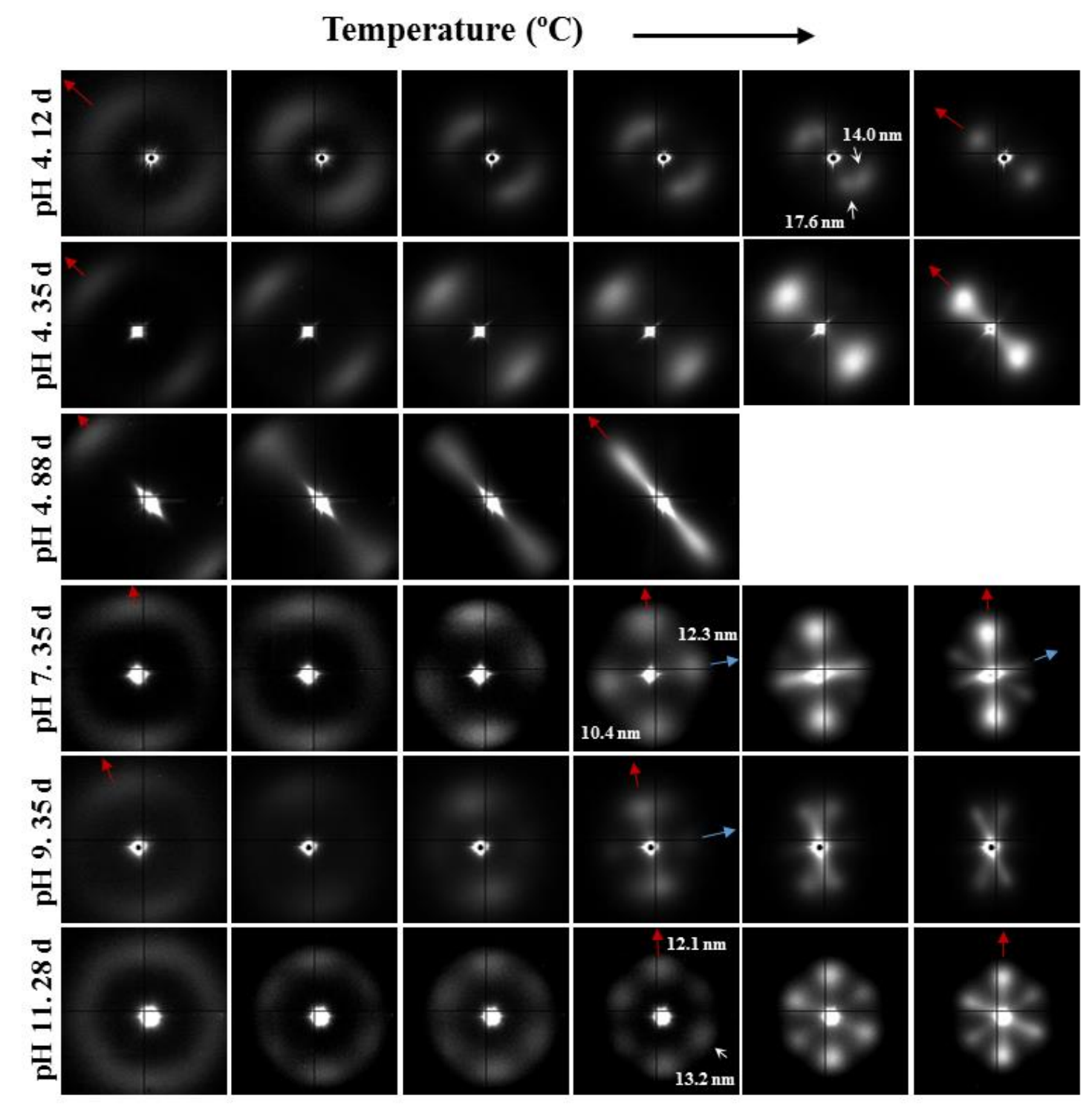

Figure 15. SAXS patterns taken during a heating run at $10{ }^{\circ} \mathrm{C} / \mathrm{min}$ of $\gamma$ irradiated GL- $b$-[GL-co-TMC-co-CL]- $b$-GL samples after being degraded in the indicated media and exposure times. Patterns in each row were acquired at increased temperatures from left to right. Red and blue arrows indicate the meridional and equatorial fiber directions.

(1)Samples degraded in acidic media basically showed the characteristic meridional reflections associated with lamellar stacking. Firstly, these reflections had the previously indicated evolution when temperature was increased that led to an increase of both lamellar thickness and breadth. However, at the highest temperatures and especially for the most degraded samples (e.g., see patterns 
of samples degraded for 35 and 88 days), reflections were elongated in the meridional direction and extended towards the center of the pattern as presumable for a decrease of the crystalline domain size (i.e., the number of stacked lamellae). It is interesting to note that extrameridional reflections with a practically meridional orientation could also be detected during the heating of the less degraded samples (e.g., see arrow for the sample exposed for 12 days at $\mathrm{pH} 4$ ). These spots suggest the sporadic formation and tilting of lamellae with a slightly greater thickness (i.e., $17.6 \mathrm{~nm}$ respect to $14.0 \mathrm{~nm}$ ) that cannot be well accommodated in the surrounding and compact amorphous phase (Figure $8 b$ ). Note that solubilization of degradation products in the acidic medium was scarce at low exposure times.

(2)An equatorial reflection is enhanced during heating of samples degraded at a neutral $\mathrm{pH}$ where the development of longitudinal cracks is characteristic. This reflection can be associated to the interfibrillar spacing (Figure 8b) which is greater than the interlamellar one (e.g., ca. $10.4 \mathrm{~nm}$ respect to ca. $12.3 \mathrm{~nm}$ as can be measured in the patterns shown in Figure 15). The regularity of interfibrillar domains was lost at the highest temperatures and consequently the corresponding reflection clearly disappeared before lamellar stacking was affected by partial fusion. Figure 15 also shows as equatorial reflections could still be detected at $\mathrm{pH} 9$.

(3)Patterns of samples exposed to basic $\mathrm{pHs}$ were more complicated since solubilization of degradation products allowed a greater readjustment/reorientation of constitutive lamellae. For example, the meridional reflection was split in the samples exposed to $\mathrm{pH}$ 9, suggesting a slight tilting of lamellae according to opposite directions. Note that optical micrographs revealed the development of tortuous fiber morphologies during exposure to basic pHs (Figure 11). This lamellar tilting seems not appropriate to justify the regular six spot pattern detected at higher $\mathrm{pHs}$, being a plausible explanation the enhancement during degradation of a macrolattice arrangement where lamellar domains were disposed at different levels along the fiber axis as displayed in Figure 8.

\subsection{Change of Lamellar Parameters of Degraded GL-b-[GL-co-TMC-co-CL]-b-GL Samples during Subsequent Non-Isothermal Crystallization and Reheating Processes}

The complex lamellar structures obtained during the heating treatment of degraded fibers were not observed again after a non-isothermal crystallization from the melt state. Therefore a homogeneous lamellar morphology was inferred for the spherulitic aggregates obtained by melt crystallization. A single and strong ring in the corresponding SAXS patterns (Figure 16a) was always detected, being also possible to get details of the lamellar stacking through the analysis of the well-defined correlation functions (Figure 16b). Nevertheless, small differences were detected between samples submitted at different degrees of degradation. The lamellar stacking of the non-degraded and $\gamma$ irradiated GL- $b$-[GL-co-TMC-co-CL]- $b$-GL sample was characterized by $L_{B}, L_{\gamma}, l_{c}$, and $l_{a}$ values of $15.1,12.8$, 10.4 , and $2.4 \mathrm{~nm}$, respectively, which were clearly different from those observed for both the initial fiber and that submitted to the thermal annealing process. Specifically, long period $L_{\gamma}$ was lower than attained after annealing (i.e., $12.8 \mathrm{~nm}$ with respect to $16.8 \mathrm{~nm}$ ) as typical for a non-isothermal crystallization where lamellar insertion mechanism takes place at the ending stages due to the geometrical constraints imposed for the previously formed thicker lamellae. Note that in any case, $l_{c}$ and $l_{a}$ decreased in a proportional way giving rise to a similar $X_{c}{ }^{S A X S}$ crystallinity (i.e., 0.81). 
a)

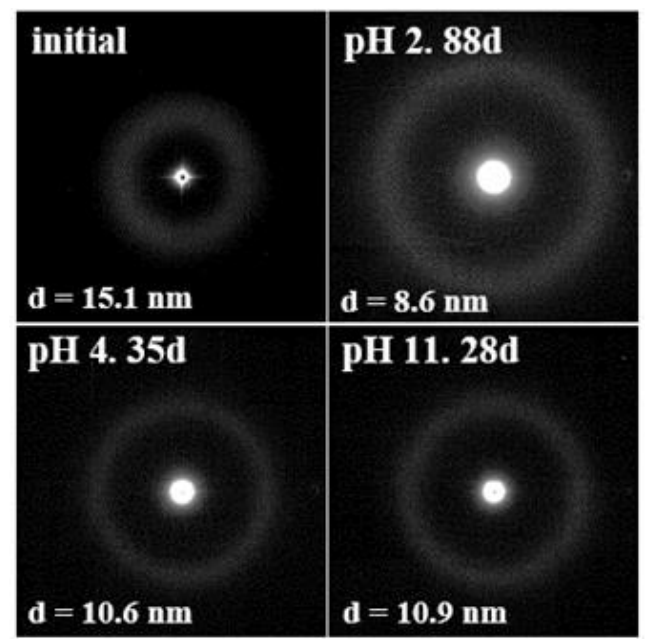

b)

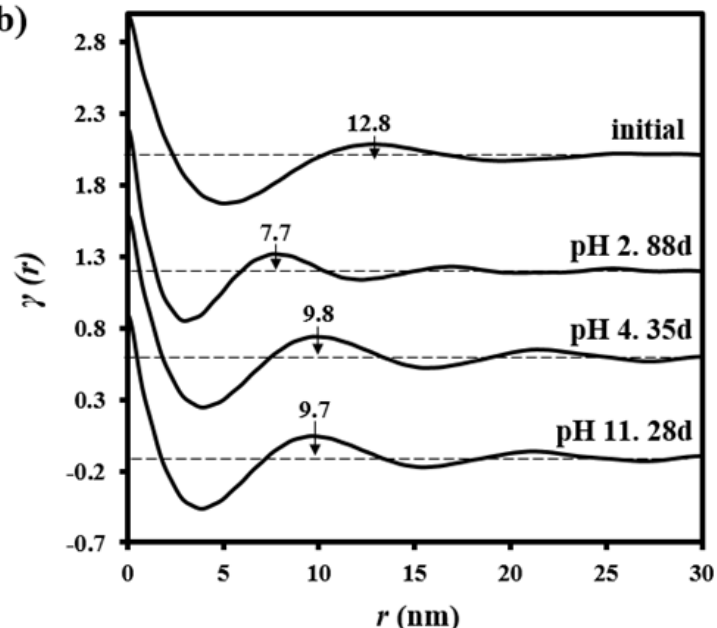

c)

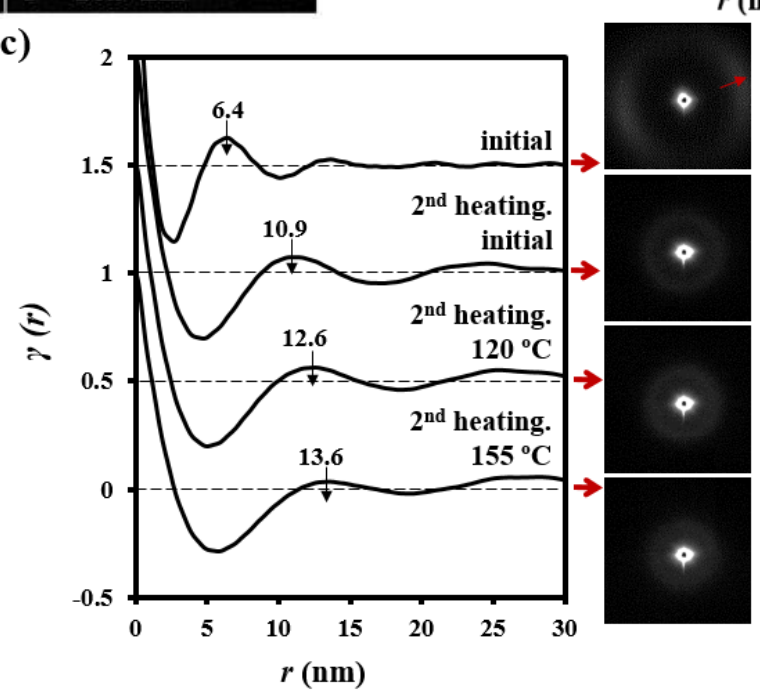

Figure 16. SAXS patterns (a) and corresponding correlation functions (b) at the end of a cooling run performed at $10{ }^{\circ} \mathrm{C} / \mathrm{min}$ from the melt state of $\gamma$ irradiated GL- $b$-[GL-co-TMC-co-CL]- $b$-GL samples before and after being degraded at $\mathrm{pH} 4$ for 35 days, $\mathrm{pH} 11$ for 28 days and $\mathrm{pH} 2$ for 88 days. (c) SAXS patterns and correlation functions of a $\gamma$ irradiated GL- $b$-[GL-co-TMC-co-CL]- $b$-GL sample degraded at $\mathrm{pH} 9$ for 35 days that were obtained from sample directly extracted from the degradation medium and at different temperatures during a second heating run performed at $10{ }^{\circ} \mathrm{C} / \mathrm{min}$. Red arrows indicate the fiber direction.

Lamellar spacing clearly decreased with the degree of degradation, suggesting that the insertion mechanism was favored. Note that $L_{B}$ decreased to $10.9-10.6 \mathrm{~nm}$ and $8.6 \mathrm{~nm}$ for samples submitted to degradation for 28-35 days (i.e., the time required to reach the asymptotic molecular weight at a given $\mathrm{pH})$ and 88 days, respectively. Note also that minimum changes were detected between samples exposed to different media but with similar degrees of degradation (e.g., $\mathrm{pH} 4$ for 35 days and $\mathrm{pH} 11$ for 28 days). The $X_{c}^{\text {SAXS }}$ crystallinity of all these degraded samples was kept in the $0.81-0.80$ range, obviously as a consequence of a proportional reduction of $l_{c}$ and $l_{a}$. Therefore, the smaller and even more regular molecular fragments (i.e., those enriched on polyglycolide hard blocks) formed during degradation did not cause a significant variation of $X_{c}^{S A X S}$ crystallinity. 
Subsequent heating runs demonstrated the capability of degraded polymers to experiment again reordering processes that led to a lamellar thickening (Figure 16c for a representative sample degraded at $\mathrm{pH} 9$ for 35 days). Final long periods $\left(L_{\gamma}\right)$ were lower than attained with the non-degraded polymers (e.g., 13.6 with respect to $16.8 \mathrm{~nm}$ ) also in agreement with the lower spacing observed when non-isothermally crystallized samples were compared (Figure 16b). Correlation functions showed that both $l_{c}$ and $l_{a}$ values increased during the reordering process in such a way that $X_{c}{ }^{S A X S}$ was again practically constant (i.e., 0.82) and comparable to that attained with the non-degraded sample. Figure 16c also highlighted the great difference between the lamellar structure of degraded fibers and that obtained after the thermal annealing of the corresponding melt crystallized spherulites. Thus, for example, $L_{\gamma}$ drastically changed from 6.4 to $13.6 \mathrm{~nm}$.

\section{Conclusions}

Hydrolytic degradation of the segmented GL- $b$-[GL-co-TMC-co-CL]- $b$-GL copolymers took place faster after being $\gamma$ irradiated despite this treatment caused also cross-linking reactions and a slight increase of the initial molecular weight. Samples exposed to hydrolytic media experienced a quick decrease of the molecular weight until a constant value was reached but weight loss was still observed for longer exposure times. The gradual increase of the $\mathrm{pH}$ of the media caused also a progressive acceleration of degradation due to the greater solubilization of decomposition products and the modification of the hydrolysis mechanism.

Degraded fibers showed complex morphologic changes that varied according to the $\mathrm{pH}$ of the medium. These changes involved the formation of longitudinal (at $\mathrm{pHs}$ equal to or greater than 5) and circumferential (at all pHs) cracks and peeling (at pHs between 6 and 8).

Small angle X-ray scattering patterns of degraded samples indicated a fast hydrolysis of chains placed in the interlamellar domains followed by a continuous attack to the crystalline domains. Furthermore, the existence of interfibrillar amorphous domains was clearly highlighted during exposure to neutral and basic media, namely when formation of longitudinal cracks was enhanced.

Subsequent annealing processes performed with degraded fibers demonstrated the capability to experiment a reordering process that increased the lamellar thickness and also the lamellar breadth. Specifically, the evolution of patterns allowed the detection of changes associated with lamellar tilting and structural features such as the presence of interfibrillar domains and the disposition of lamellar crystals at different levels along the fiber axis for a given cross-section.

Degraded samples were able to crystallize from the melt giving rise to aggregates with a lamellar structure that was clearly distinct from that observed in the initial fibers. Furthermore, differences were also detected between samples submitted at different levels of degradation. Specifically, lamellar thickness decreased when the hydrolytic attack was more aggressive as determined for both non-isothermally crystallized samples before and after a subsequent thermal annealing process.

\section{Acknowledgments}

Authors are in debt to supports from MINECO and FEDER (MAT2012-36205) and the Generalitat de Catalunya (2014SGR188). The work has also been carried out under a research agreement between B. Braun Surgical, S.A. and the Universitat Politècnica de Catalunya. Yolanda Márquez thanks financial 
support from B. Braun Surgical, S.A. Diffraction experiments were performed at NCD beamline at ALBA Synchrotron with the collaboration of ALBA staff.

\section{Author Contributions}

Yolanda Márquez performed the experiments. Juan Carlos Martínez collaborated in the acquisition and analysis of synchrotron data. Pau Turon, Lourdes Franco and Jordi Puiggalí directed the research. Jordi Puiggalí wrote the manuscript. All authors discussed the results and edited the manuscript.

\section{Conflicts of Interest}

The authors declare no conflict of interest.

\section{References}

1. Middleton, J.C.; Tipton, A.J. Synthetic biodegradable polymers as orthopedic devices. Biomaterials 2000, 21, 2335-2346.

2. Griffin Lewis, O.; Fabisial, W. Sutures. In Kirk-Othmer Encyclopedia of Chemical Technology, 4th ed.; Wiley: New York, NY, USA, 1997.

3. Gilding, D.K.; Reed, A.M. Biodegradable polymers for use in surgery-polyglycolic-poly(lactic acid) homopolymers and copolymers. Polymer 1979, 2, 1459-1464.

4. Homsy, C.A.; Mcdonald, E.R.; Akers, W.W. Surgical suture-canine tissue interaction for six common suture types. J. Biomed. Mater. Res. 1968, 2, 215-230.

5. Blomstedt, B.; Ostenberg, B. Suture materials and wound infection. An experimental study. Acta Chir. Scand. 1978, 144, 269-274.

6. Rodeheaver, G.T.; Thacker, J.G.; Owen, J.; Strauss, M.; Masterson, T.; Edlich, R.F. Knotting and handling characteristics of coated synthetic absorbable sutures. J. Surg. Res. 1983, 35, 525-530.

7. Katz, A.R.; Mukherjee, D.P.; Kaganov, A.L.; Gordon, S. A new synthetic monofilament absorbable suture made from polytrimethylene carbonate. Surgery Gynecol. Obstet. 1985, 161, 213-222.

8. Zurita, R.; Puiggalí, J.; Franco, L.; Rodríguez-Galán, A. Copolymerization of glycolide and trimethylene carbonate. J. Polym. Sci. Part A Polym. Chem. 2005, 44, 993-1013.

9. Kasperczyk, J. Copolymerization of glycolide and $\varepsilon$-caprolactone, 1: Analysis of the copolymer microstructure by means of ${ }^{1} \mathrm{H}$ and ${ }^{13} \mathrm{C}-\mathrm{NMR}$ spectroscopy. Macromol. Chem. Phys. 1999, 200, 903-910.

10. Díaz-Celorio, E.; Franco, L.; Rodríguez-Galán, A.; Puiggalí, J. Synthesis of glycolide/trimethylene carbonate copolymers: Influence of microstructure on properties. Eur. Polym. J. 2012, 48, 60-73.

11. Noorsal, K.; Mantle, M.D.; Gladden, L.F.; Cameron, R.E. Degradation and drug-release studies of a poly(glycolide-co-trimethylene carbonate) copolymer (Maxon). J. Appl. Polym. Sci. 2005, 95, 475-486.

12. Díaz-Celorio, E.; Franco, L.; Rodríguez-Galán, A.; Puiggalí, J. Study on the hydrolytic degradation of glycolide/trimethylene carbonate copolymers having different microstructure and composition. Polym. Degrad. STable 2013, 98, 133-143. 
13. Freudenberg, F.; Rewerk, S.; Kaess, M.; Weiss, C.; Dorn-Beinecke, A.; Post, S. Biodegradation of absorbable sutures in body fluids and pH buffers. Eur. Surg. Res. 2004, 36, 376-385.

14. Farrar, D.; Gillson, R. Hydrolytic degradation of polyglyconate B: the relationship between degradation time, strength and molecular weight. Biomaterials 2002, 23, 3905-3912.

15. Ahmed, A.H.; Goldie, B.S. Comparison of the mechanical properties of polyglycolide-trimethylene carbonate (Maxon) and polydioxanone suture (PDSII) used for flexor tendon repair and active mobilization. J. Hand Surg. Am. 2002, 27B, 329-332.

16. Mäkelä, P.; Pohjonen, T.; Törmalä, P.; Waris, T.; Ashammakhi, N. Strength retention properties of self-reinforced poly L-lactide (SR-PLLA) sutures compared with polyglyconate (Maxon ${ }^{\circledR}$ ) and polydioxanone (PDS) sutures. An in vitro study. Biomaterials 2002, 23, 2587-2592.

17. Díaz-Celorio, E.; Franco, L.; Puiggalí, J. Isothermal crystallization Study on a biodegradable segmented copolymer constituted by glycolide and trimethylene carbonate units. J. Appl. Polym. Sci. 2010, 116, 577-589.

18. Díaz-Celorio, E.; Franco, L.; Puiggalí, J. Nonisothermal Crystallization behavior of a biodegradable segmented copolymer constituted by glycolide and trimethylene carbonate units. J. Appl. Polym. Sci. 2011, 119, 1548-1559.

19. Tomihata, K.; Suzuki, M.; Ikada, Y. The pH dependence of monofilament sutures on hydrolytic degradation. J. Biomed. Mater. Res. 2001, 58, 511-518.

20. Díaz-Celorio, E.; Franco, L.; Puiggalí, J. Influence of microstructure on the crystallization of segmented copolymers constituted by glycolide and trimethylene carbonate units. Express Polym. Lett. 2013, 7, 186-198.

21. Oberhoffner, S.; Planck, H. Surgical Suture Material from Triblockterpolymer, Its Use in Surgery and Process for Its Preparation. EP 0835895, 3 December 2003.

22. Barber, F.A. Resorbable fixation devices: A product guide. Orthop. Spec. Ed. 1998, 4, 1111-1117.

23. Roby, M.S.; Bennet, S.L.; Liu, E.K. Absorbable Block Copolymers and Surgical Articles Fabricated Thereform. U.S. Patent 5.403.347, 4 April 1995.

24. Pineros-Fernández, A.; David, B. Drake, D.B.; Rodeheaver, P.A.; Moody, D.L.; Edlich, R.; Rodeheaver, G.T. CAPROSYN*, another major advance in synthetic monofilament absorbable suture. J. Long. Term. Eff. Med. Implants 2004, 14, 359-368.

25. Martínez-Barrera, G.; Brostow, W. Fiber-reinforced polymer concrete: Property improvement by gamma irradiation. In Gamma Radiation Effects on Polymeric Materials and Its Applications; Barrera, C., Ed.; Research Signpost: Kerala, India, 2009; pp. 27-44.

26. D'Alelio, G.F.; Haberli, R.; Pezdirtz, G.F. Effect of ionizing radiation on a series of saturated polyester. J. Macromol. Sci. Chem. 1968, 2, 501-588.

27. Zhang, L.; Loh, I.H.; Chu, C.C. A combined gamma irradiation and plasma deposition treatment to achieve that ideal degradation properties of synthetic absorbable polymers. J. Biomed. Mater. Res. 1993, 27, 1425-1441. 
28. Chu, C.C.; Williams, D.F. The effect of gamma irradiation on the enzymatic degradation of polyglycolic acid absorbable sutures. J. Biomed. Mater. Res. 1983, 17, 1029-1040.

29. Márquez, Y.; Franco, L.; Turon, P.; Rodríguez-Galán, A.; Puiggalí, J. Study on the hydrolytic degradation of the segmented GL- $b$-[GL-co-TMC- $c o-\mathrm{CL}]-b-\mathrm{GL}$ copolymer with application as monofilar surgical suture. Polym. Degrad. STable 2013, 98, 2709-2721.

30. Theorell, T.; Stenhagen, E. Ein Universalpuffer fiir den pH-Bereich 2.0 bis 12.0. Biochem. Z. 1939, 299, 416-419. (In German)

31. Lin, H.L.; Chu, C.C.; Grubb, D. Hydrolytic degradation and morphologic study of poly-p-dioxanone. J. Biomed. Mater. Res. 1993, 27, 153-166.

32. McIntyre, J.E. Polyester Fibres. In Handbook of Fibre and Technology: Fibre Chemistry; Lewin, M., Pearce, E.M., Eds.; Marcel Dekker, Inc.: New York, NY, USA, 1998; pp. 1-73.

33. Li, S.M. Hydrolytic degradation characteristics of aliphatic polyesters derived from lactic and glycolic acids. J. Biomed. Mater. Res. Part B Appl. Biomater. 1999, 48B, 342-353.

34. Fischer, E.W.; Sterzel, H.J.; Wegner, G. Investigation of the structure of solution grown crystals of lactide copolymers by means of chemical reactions. Kolloid Z. Z. Polym. 1973, 251, 980-990.

35. Fredericks, R.J.; Melveger, A.J.; Dolegiewtz, L.J. Morphological and structural changes in a copolymer of glycolide and lactide occurring as a result of hydrolysis. J. Polym. Sci. Polym. Phys. Ed. 1984, 22, 57-66.

36. Wu, L.; Ding, J. Effects of porosity and pore size on in vitro degradation of three-dimensional porous poly(D,L-lactide-co-glycolide) scaffolds for tissue engineering. J. Biomed. Mater. Res. A 2005, 75, 767-777.

37. Fernández, J.; Larrañaga, A.; Etxeberria, A.; Sarasua, J.R. Effects of chain microstructures and derived crystallization capability on hydrolytic degradation of poly(L-lactide/E-caprolactone) copolymers. Polym. Degrad. Stab. 2013, 98, 481-489.

38. Kulkarni, A.; Reiche, J.; Lendlein, A. Hydrolytic degradation of poly(rac-lactide) and poly[(rac-lactide)-co-glycolide] at the air water interface. Surf. Interface. Anal. 2007, 39, 740-746.

39. Yoshioka, T.; Kawazoe, N.; Tateishi, T.; Chen, G. In vitro evaluation of biodegradation of poly(lactic-co-glycolic acid) sponges. Biomaterials 2008, 29, 3438-3443.

40. Peterlin, A. Morphology and properties of crystalline polymers with fiber structure. Text. Res. J. 1972, 42, 20-30.

41. Murthy, N.S.; Reimschuessel, A.C.; Kramer, V. Changes in void content and free volume in fibers during heat setting and their influence on dye diffusion and mechanical properties. J. Appl. Polym. Sci. 1990, 40, 249-262.

42. Chatani, Y.; Suehiro, K.; Okita, Y.; Tadokoro, H.; Chujo, K. Structural studies of polyesters. I Crystal structure of polyglycolide. Die Makrornolekulare Chem. 1968, 113, 215-229.

43. Vonk, C.G.; Kortleve, G. X-ray small-angle scattering of bulk polyethylene. Kolloid Z. Z. Polym. 1967, 220, 19-24.

44. Vonk, C.G. A general computer program for the processing of small-angle X-ray scattering data. J. Appl. Crystallogr. 1975, 8, 340-341. 
45. Hsiao, B.S.; Verma, R.K. A novel approach to extract morphological variables in crystalline polymers from time-resolved SAXS measurements. J. Synchrotron Radiat. 1998, 5, 23-29.

46. Stribeck, N. Extraction of domain strucure information from small angle scattering patterns of bulk materials. J. Appl. Crystallogr. 2001, 34, 496-503.

47. Stribeck, N.; Fakirov, S. Three-dimensional chord distribution function SAXS analysis of the strained domain structure of a poly(ether ester) thermoplastic elastomer. Macromolecules 2001, 34, 7758-7761.

(C) 2015 by the authors; licensee MDPI, Basel, Switzerland. This article is an open access article distributed under the terms and conditions of the Creative Commons Attribution license (http://creativecommons.org/licenses/by/4.0/). 